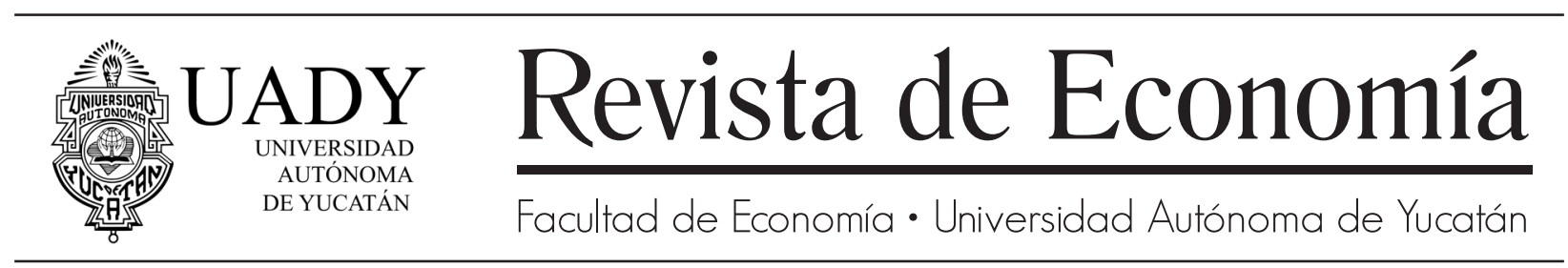

Valoración económica de la multifuncionalidad de los parques urbanos: un análisis explicativo - Economic valuation of the multifunctionality of urban parks: an explanatory analysis

Diego Adiel Sandoval Chávez ${ }^{1}$ Ana Córdova y Vázquez ${ }^{2}$ Esmeralda Cervantes Rendón ${ }^{3}$

\title{
Resumen
}

Luis Ernesto Cervera Gómez ${ }^{4}$ Aida Yarira Reyes Escalante ${ }^{5}$

Los parques urbanos brindan una gran variedad de beneficios ambientales, sociales y económicos. El valor de estos beneficios no se considera y a menudo los estudios lo pasan por alto. En la búsqueda de documentos relacionados con la valoración económica de los parques urbanos en bases de datos y motores de búsqueda, no fue posible encontrar un análisis explicativo de las características generales de los modelos de valoración económica aplicables a los parques urbanos. El objetivo de este documento es realizar tal esfuerzo. Mediante la realización de una investigación explicativa-descriptiva, a través de un estudio longitudinal, se analizaron 120 publicaciones en español e inglés, correspondientes a un período de 57 años (1963-2020). Las publicaciones se clasificaron en modelos de precios hedónicos, modelo de costos de viaje y valoración contingente. Para cada una de estas tres categorías también se consideraron las subcategorías de teoría primaria, teoría secundaria, opinión, revisión y caso de estudio. El objetivo era analizar y comprender el contexto y las circunstancias en que se llevaron a cabo los estudios y evaluar su relevancia, fortalezas y debilidades. Este enfoque llevó a identificar cuáles modelos todavía están en uso y cómo se adoptan. El modelo de precios hedónicos utilizando el formato de regresión semi-logarítmica es el más utilizado para la valoración, principalmente en los Estados Unidos y Europa. Se puede concluir que la valoración económica de los parques urbanos es un tema de interés global debido a su contribución a la sustentabilidad en el ámbito urbano.

Palabras clave: valoración económica, modelo de precios hedónicos, modelo del costo del viaje, valoración contingente, parques urbanos.

Clasificación JEL: Q5

1- Tecnológico Nacional de México Campus Ciudad Juárez, México. Correo electrónico: dsandoval@itcj.edu.mx

(D)ORCID: https://orcid.org/0000-0002-2536-1844

2- El Colegio de la Frontera Norte, México. Correo electrónico: acordova@colef.mx

(D)ORCID: https://orcid.org/0000-0003-4551-5123

3- El Colegio de Chihuahua, México. Correo electrónico: ecervantes@colech.edu.mx

(D)ORCID: https://orcid.org/0000-0003-0227-0963

4- El Colegio de Chihuahua, México. Correo electrónico: Icervera@colechedu.onmicrosoft.com

(D)ORCID: https://orcid.org/0000-0003-0495-7590

5- Universidad Autónoma de Ciudad Juárez, México. Correo electrónico: aida.reyes@uacj.mx

(D)ORCID: https://orcid.org/0000-0002-0104-9522

RECEPCIÓN: 5 de julio de 2020 ACEPTACIÓN: 20 de octubre de 2020 REVISTA DE ECONOMÍA: Vol. 38- Núm 96 ENERO A JUNIO DE 2021: Págs. 89-119

e-ISSN: 2395-8715

http://www.revista.economia.uady.mx/index.php/reveco

Esta obra está bajo una licencia de Creative Commons Atribución-NoComercial-Compartirlgual 4.0 Internacional. (CC BY-NC-SA 4.0)

http://creativecommons.org/licenses/by-nc-sa/4.0 


\section{$\searrow$ Abstract}

Urban parks provide a great variety of environmental, social and economic benefits. The value of these benefits is elusive and oftentimes studies overlook it. In a search for urban park economic valuation-related papers in databases and search engines, an explanatory analysis of the overall characteristics of the economic valuation models applicable to urban parks could not be found. The objective of this work is to perform such an endeavor. By conducting an explanatory-descriptive research, through a longitudinal study, 120 publications in the English and Spanish languages, corresponding to a period of 57 years (1963-2020) were analyzed. Publications were categorized in hedonic price model, travel cost model, and contingent value. For each of these three schemes the subcategories primary theory, secondary theory, opinion, review and case study were also considered. The aim was to analyze and understand the context and circumstances in which they were conducted, and to evaluate their relevance, strengths, and weaknesses. This approach led to realize what models are still in use and how they are adopted. The hedonic pricing model using the semi-logarithmic regression format is the most widely used for valuation, mainly in the United States and Europe. In can be concluded that the economic valuation of urban parks is a subject of global interest due to its contribution to attain sustainability in the urban realm.

Keywords: economic valuation, hedonic price model, cost of travel model, contingent valuation, urban parks.

JEL Classification: Q5

\section{Introducción}

Los parques urbanos juegan un rol crucial en la sustentabilidad de las ciudades porque cumplen una gran diversidad de funciones sociales, medioambientales y económicas. En el espacio público las personas se reconocen como parte de una comunidad, y en este sentido, los parques son quizás la forma más depurada de espacio público porque tienen la capacidad de desarrollar los sentimientos de amor por la vida o biofilia (Wilson, 1984). Los parques amalgaman aspiraciones colectivas en la medida en la que las personas pueden apropiarse del espacio. Esta apropiación da lugar a un mayor y mejor capital social, ya sea creándolo, reforzándolo o inclusive solo manteniéndolo (Urteaga, 2013). Los parques son los grandes articuladores de la dinámica social, escenifican la diversidad cultural, rompen brechas generacionales, cierran abismos de marginalidad, empoderan a los vulnerables, promueven encuentros e intercambios, además de generar valores simbólicos y sentidos de identidad y pertenencia (Borja, 2011; Silva, 2006).

Córdova y Martínez-Soto (2014) dan cuenta de los beneficios que brindan los parques urbanos, entre los que se encuentran, desde el punto de vista social, la recreación y el esparcimiento. Estos dos servicios, junto con la estética de la vegetación y las amenidades, distraen la mente del estrés y brindan tranquilidad, lo que ayuda a prevenir los padecimientos mentales. A su vez, en los parques se practica ejercicio, lo que ayuda a disminuir la obesidad y el síndrome metabólico, además de prevenir los padecimientos cardiovasculares. Desde el punto de vista ambiental, los parques desempeñan diversas funciones: purifican el aire filtrando 
contaminantes, oxigenan el aire, capturan $\mathrm{CO}_{2}$ y lo depositan en sus cuerpos leñosos, capturan material particulado, interceptan escorrentías, entre otros muchos.

Todos los beneficios que se han mencionado se derivan de las características que distinguen a los parques urbanos. Los parques son bienes públicos no comercializables a los que se hace referencia como bienes no consumibles, de no mercado, o no basados en el mercado. Al valor económico de estos bienes se le conoce como valor de no mercado (VNM) (Choumert y Salanié, 2008). De acuerdo con Tietenberg y Lewis (2012), el enfoque clásico de la economía ambiental es abordar el VNM del parque desde dos grandes avenidas: valor de uso (o valor consuntivo) y valor de no uso (o valor no consuntivo). Es posible expresar el valor del uso del espacio en directo, indirecto y opcional. El valor de uso directo se refiere a la utilidad percibida al acudir al espacio, esto es, al ocupar un lugar y disponer de sus características o amenidades (la asistencia a un parque por motivos de recreación o esparcimiento, por ejemplo). El valor de uso indirecto se refiere al valor de los beneficios del espacio que son aprovechados por las personas, sin que implique un uso o estancia en el lugar (tales como los beneficios ambientales, como la purificación del aire, por ejemplo). El valor de uso opcional refiere a tener la capacidad de utilizar el parque a voluntad dentro de las restricciones de operación (una membresía o el hecho que se pueda asistir al espacio a voluntad, por ejemplo). Por otra parte, el valor de no uso se refiere al valor de la existencia misma del parque y a su valor de legado, lo que el espacio simboliza y que hace que las personas estén dispuestas a donar o a participar con su trabajo para la permanencia del mismo. La idea central se basa en la tesis de Lancaster (1966) quien establece que las características de un bien son las que determinan la utilidad del consumidor.

Para estimar el valor de un parque se han adoptado métodos directos en los que se les pregunta a las personas sus preferencias y el valor que le asignan al espacio. En esta categoría se ubica la Valoración Contingente (VC) (Darling, 1973). Mediante una entrevista, se le pregunta a la persona la disposición máxima a pagar al mostrarle el escenario de un mercado hipotético. En este caso, se trata de un conjunto de preferencias establecidas por el individuo cuestionado. Por otra parte, los métodos indirectos utilizan mercados sustitutos para estudiar las preferencias de las personas (Choumert y Salanié, 2008). Los dos principales esquemas de valoración indirecta son el Modelo de Precios Hedónicos (MPH) y El Modelo del Costo del Viaje (MCV). En el MPH se busca estimar el impacto que tiene en el valor de las propiedades la cercanía con un parque (Rosen, 1974). Por su parte, en el MCV se busca estimar el valor del espacio relacionándolo con los costos de traslado, el precio de entrada y el valor del tiempo que se invierte visitándolo (Cesario, 1976).

Los tres anteriores esquemas son los que, desde el enfoque de la disciplina de la economía, se han adoptado con mayor frecuencia para establecer una estimación del VNM a un bien público, como lo es un parque urbano. Lo anterior permite establecer como objetivo general de este trabajo el determinar los enfoques de aplicación de los modelos de valoración económica de la multifuncionalidad de los parques urbanos.

En el artículo primero se revisan los fundamentos teóricos de los modelos económicos MPH, MCV y VC; después, se presenta el método utilizado, que consistió en una revisión explicativa de la literatura. A lo anterior le sigue la caracterización de la aplicación de estos modelos en relación con los parques urbanos, revisando los fundamentos que les dieron origen, para después 
poner énfasis en la frecuencia y contexto de aplicación de cada modelo. Por último, luego de una discusión detallada de los hallazgos, se presentan las conclusiones que del trabajo se derivan.

\section{Modelos económicos de valoración de parques urbanos}

\subsection{Modelo de Precios Hedónicos (MPH)}

El MPH busca reflejar los beneficios de los parques urbanos en el precio de las viviendas. Se parte del hecho que dichos beneficios no son capitalizados en el valor de mercado de las viviendas, es decir, se trata de externalidades por las cuales el propietario no hace un desembolso explícito. El punto de inicio de este modelo se le atribuye mayoritariamente a Griliches (1971) y a Rosen (1974), quienes ponen los cimientos de la teoría y aportan el marco metodológico para su aplicación. Sin embargo, Weigher y Zerbst (1973) afirman que el concepto pudiera haberse originado a raíz del trabajo de Friedman y Friedman (1962), donde se trata de justificar la inversión en parques urbanos haciendo referencia a las externalidades que benefician a los no usuarios. Por otra parte, Colwell y Dilmore (1999) se dieron a la tarea de tratar de dar certeza acerca del origen del MPH. De acuerdo con ellos, el origen pudiera remontarse al trabajo de Lancaster (1966) quien dio la pauta para considerar el valor de un bien sobre la base de sus características y peculiaridades, y no por su valor intrínseco. Entonces, el MPH intenta estimar el VNM, es decir, de uso no consumible, que se refleja en la disposición a pagar por los servicios que brinda un bien, en nuestro caso un parque urbano.

Mediante un modelo de regresión econométrico se asocia el valor de mercado de las viviendas con las características estructurales (terreno, habitaciones, tipo, entre otros) y con las de localización (características del vecindario, tales como: distancia al centro de trabajo, distancia al parque, entre otros). De esta manera, el precio de la vivienda está en función de una variedad de atributos y características. En el contexto de la existencia de un parque urbano, se tiene:

$$
P=f\left(A_{i}, L_{j}, E_{k}\right)
$$

Donde $A_{1}$ es el vector de las características estructurales, $L_{j}$ es el vector de los atributos de localización y $E_{k}$ es el vector de las características del parque. Si se deriva parcialmente $P$ con respecto a una característica de $E_{k}$, se obtiene el precio implícito en relación con esa característica. En un segundo análisis, este precio implícito se relaciona con otras características de interés, estimándose así las valoraciones que al efecto se busquen. El MPH revela la disposición a pagar por estar cerca de un parque (distancia), por las dimensiones del mismo (área), o por cualquier otra variable de interés relacionada con el parque.

El modelo de regresión no está exento de problemas en la práctica. Cho, Bowker y Park (2006) enumeran una serie de dificultades al respecto: (a) alta heterogeneidad del mercado de viviendas, lo que dificulta la estimación de factores individuales; (b) multicolinealidad y heterocedasticidad, lo que viola los supuestos del modelo estadístico; (c) dependencia espacial 
por la presencia de otro atractivo; (d) interrelaciones con otros mercados o falta de conocimiento a priori; (e) limitaciones en la disponibilidad de los datos; (f) no siempre se cumple la condición de equilibrio. Para hacer frente a las dificultades del modelo estadístico se ha recomendado utilizar la transformación Box-Cox, además de los formatos semilogarítmico y logarítmico, principalmente, aunque se han adoptado también otros modelos no lineales (Cropper, Deck y McConnell, 1988).

\subsection{Modelo del costo del viaje (MCV)}

EI MCV es otra alternativa para la VNM de los parques urbanos. El objetivo es encontrar una estimación del valor de un espacio sobre la base de la utilidad que percibe un visitante al hacer un uso consuntivo de uno o más de los atractivos ambientales o de recreación (Cesario, 1976). EI MCV pone atención en valorar dos conceptos: (a) el desembolso que el visitante incurre al trasladarse desde un origen hasta el espacio en cuestión, y (b) el costo de oportunidad en el que incurre el visitante para acudir al lugar (Willis y Garrod, 1991). Es fundamental determinar la demanda en un cierto periodo de tiempo, así como el valor del tiempo de traslado y costo de oportunidad incurrido. Con esta información, es posible establecer una estimación del valor de los servicios que presta el espacio en dicho periodo. En el MCV se asume que, en una visita de propósito único y sin que exista un sitio sustituto con atributos similares, los visitantes maximizan su utilidad sujetándola a restricciones de ingreso y tiempo. Se asume también que los tiempos de trabajo y permanencia en el lugar son irrestrictos (Osorio y Correa, 2004). Existe consenso en la literatura acerca de que el MCV se inició con el trabajo pionero de Hotelling (1949), el subsiguiente de Cesario (1976) y recientemente el de Clawson \& Knetsch (2013).

Siguiendo a Martínez-Cruz (2005), la estructura general del MCV es la siguiente:

$$
\begin{aligned}
& \operatorname{Max}: U(X, r, q) \\
& \text { Sujeto a: } \\
& M+\left(p_{w} * t_{w}\right)=X+(c * r) \\
& t^{*}=\left(t_{1}-t_{2}\right) r
\end{aligned}
$$

Donde $U$ es la función de utilidad a maximizar; $X$ es la cantidad de numerario a cierto precio; $r$ es el número de visitas al espacio; $q$ es el nivel de calidad del espacio (ambiental, recreación, entre otros); $M$ es el ingreso exógeno; $p_{w}$ es la tasa salarial; $c$ es el costo monetario de una visita; $t$ es el tiempo disponible; $t_{w}$ son las horas trabajadas; $t_{1}$ es el tiempo de traslado en viaje redondo; $t_{2}$ es el tiempo de permanencia en el espacio.

Las dificultades que enfrenta el MCV en su aplicación son: (a) frecuente violación a la condición de homogeneidad de los visitantes; (b) no siempre es posible asumir que el costo del viaje tiene solo dos componentes; (c) no es fácil de modelar estadísticamente; (d) no siempre se discrimina a los visitantes recurrentes; (e) imprecisiones por falta de conocimiento a priori del espacio (Martínez-Cruz y Sainz, 2017). El conteo de clases latentes, el uso de distribuciones de 
probabilidad alternativas, la trasformación Box-Cox, así como los refinamientos y mejores pilotajes de las encuestas han permitido solventar estas dificultades (Bartczak et al., 2008; Martínez-Cruz, 2005; Tyrväinen y Miettinen, 2000; Varela, Börjesson y Daly, 2018; Sánchez, 2008).

\subsection{Valoración contingente (VC)}

El modelo VC tiene la particularidad que, según sea el caso, usuarios y no usuarios declaran sus deseos y preferencias en relación con los atractivos y amenidades de un determinado espacio. El desarrollo conceptual de la VC se le atribute a Davis (1963), quien a su vez se fundamentó en los conceptos de valoración de bienes no basados en el mercado, particularmente el uso directo de los servicios de recreación al aire libre. Por otra parte, el trabajo de Darling (1973), para valorar los beneficios de un conjunto de parques acuáticos urbanos, es considerado el punto de partida en la adopción de la VC.

El instrumento utilizado en la VC es un cuestionario en el que se les sugiere a las personas de interés un escenario hipotético para identificar la eventual existencia de valores monetarios similares a la oferta ambiental o de recreación que se ofrece en el mercado tradicional. Se les pregunta directamente acerca de la cantidad que estarían dispuestos a pagar por un bien público, ya sea por sus beneficios directos (uso consuntivo) o por su simple existencia (uso pasivo) (Carson, Flores y Meade, 2001). Existen varios enfoques a la hora de diseñar el formato de pregunta: opción múltiple, tarjeta de opciones, subasta, declaración espontánea y dicotómica. El que ha brindado mejores resultados es el formato dicotómico (Arrow et al., 1993).

La mecánica de la VC (Cerda, Riofrio y Vial, 1997) establece que la probabilidad que la persona responda afirmativamente a la cantidad sugerida es igual a la probabilidad que el diferencial de utilidad estimada $(\Delta V)$ sea mayor que el término $\eta$, esto es:

$$
P(s i)=P[\Delta V>\eta]=F[\Delta V]=\int_{-\infty}^{\Delta V} f(\eta) d \eta
$$

Al igual que todo modelo estadístico, la VC se enfrenta a dificultades y obstáculos cuando se adopta en la práctica. La mayor dificultad se presenta a la hora de levantar la encuesta. En este sentido, Cristeche y Penna (2008) advierten que deben tomarse ciertas precauciones al aplicar los cuestionarios: (a) elegir adecuadamente el formato de la pregunta; (b) contabilizar y discriminar las preguntas negativas (preguntas cero); (c) disminuir el sesgo en las preguntas; (d) reconocer el recelo a por valorar bienes públicos; (e) identificar y discriminar respuestas deshonestas o no objetivas; ( $f$ ) desconocimiento del sitio a evaluar. Para lidiar con estas dificultades es muy importante elegir el marco econométrico adecuado y pilotear los cuestionarios, privilegiando las respuestas dicotómicas.

\section{Aplicación de los modelos económicos de valoración de parques urbanos}

La aplicación de los modelos económicos de valoración de los parques (modelo de precios hedónicos, modelo del costo del viaje y valoración contingente) constituyen la visión económica clásica de la valoración de bienes no consumibles o no basados en el mercado. 
Los modelos se encuentran ubicados en tres corrientes de aplicación: teoría primaria, teoría secundaria, reporte corporativo y estudio de caso. A su vez, los estudios de caso se analizaron en las categorías: país, escala, modelo estadístico, criterio y valor encontrado.

La aplicación de los modelos que se encuentran dentro de teoría primaria se consideran puntos de partida de la categoría y son citados reiteradamente como tales. Los trabajos considerados en la teoría secundaria son aquellos que se desviaron hacia otra forma de valoración, pero permaneciendo dentro del esquema de la teoría primaria. La aplicación de los modelos fuera del sector académico se les considera reportes corporativos y, por último, las aplicaciones específicas se clasificaron como estudios de caso.

Dentro de los datos de aplicación de los modelos se pueden identificar datos generales como: país de procedencia, escala de aplicación, modelo estadístico adoptado, criterio utilizado y valor encontrado. Las aplicaciones de los tres modelos: MPH, MCV y VC han sido documentadas en 120 publicaciones en un periodo de 57 años (1963-2020), con una referencia a un trabajo de 1949. Se encontraron 66 publicaciones relativas al MPH (55\%), 14 correspondientes al MCV (11.67\%) y 40 pertenecientes a la VC (33.33\%).

Las aplicaciones de los modelos dependen de la región, en Estados Unidos se cuenta con un total de 42 publicaciones (35\%), Europa 35 trabajos (29.17\%), Asia y Oceanía 20 (16.67\%), Latinoamérica 20 (16.67\%) y África $3(2.50 \%)$. En Estados Unidos y Europa, se originaron la gran mayoría de las publicaciones con 77 (64.17\%).

De acuerdo a las aplicaciones de los modelos, se ha observado un interés constante, sobre todo en las dos últimas décadas, ver Gráfica 1. Dentro de las aplicaciones de las teorías primarias se cuenta con un total de $7(5.83 \%)$, una teoría secundaria $(0.86 \%)$, dos publicaciones de opinión (1.67\%), 11 trabajos de revisión (9.17\%), un reporte corporativo $(0.83 \%)$ y 98 estudios de caso (81.76\%), siendo este último es más utilizado.

Gráfica 1. Aplicaciones de los modelos de valoración económica de parques urbanos

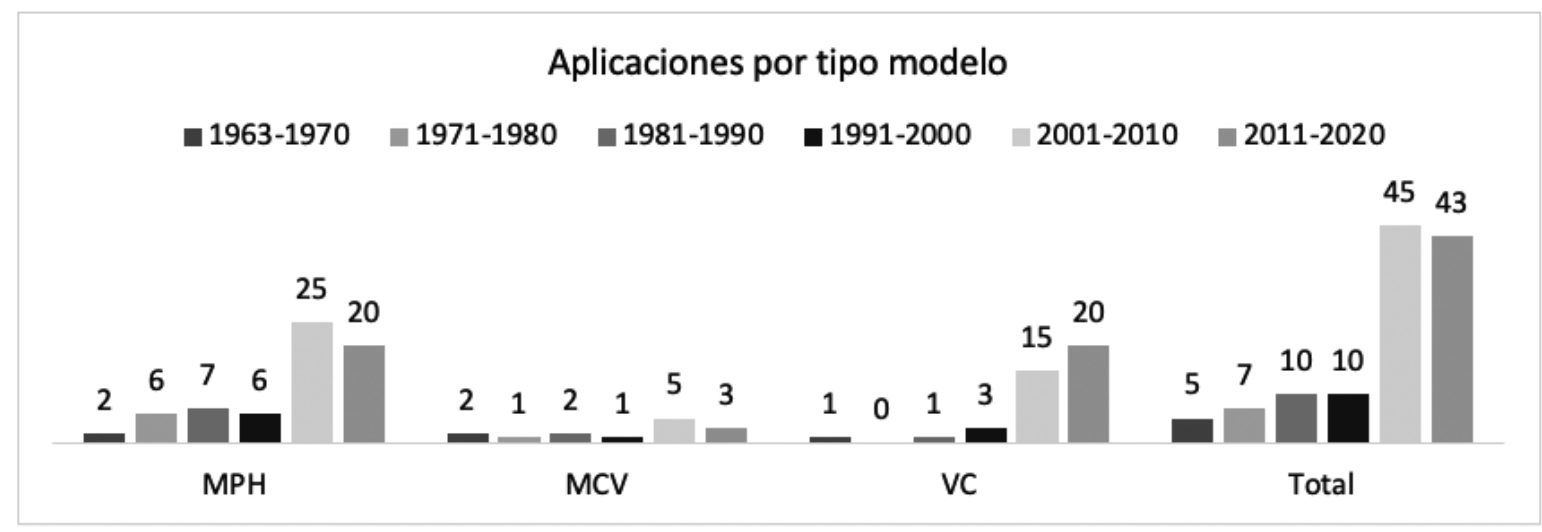

Fuente: elaboración propia 


\section{Método}

El encuadre metodológico establece un estudio descriptivo explicativo de los modelos de valoración económica. Para tal análisis se consideró una población de 120 artículos de aplicación que fueron publicados en diversas bases de datos. Se considera un estudio longitudinal ya que se tomó el periodo comprendido entre 1963 a diciembre de 2020, se condujo una búsqueda en los principales motores académicos en los idiomas inglés y español, tales como: ScienceDirect, Google Scholar, Google, Scielo, Redalyc, Dialnet, EconPapers, Springer Link, World Wide Seek. Para el estudio se establecen tres momentos de estudio:

1. Búsqueda de publicaciones utilizando las palabras claves relativas a la valoración de: parques urbanos, espacios verdes, áreas verdes, naturaleza urbana, jardines urbanos, huertos urbanos. En idioma inglés: valuation of urban parks, open spaces, urban greenspace, green space. Una vez identificados los principales temas, se utilizaron indicadores identificados como las palabras claves derivadas: modelo del costo del viaje, valoración contingente, precios hedónicos, beneficios económicos de los parques. En idioma inglés: travel cost method, contingent valuation, hedonic prices, and economic benefits of urban greenspaces.

2. Identificación de los enfoques utilizando los modelos MPV, MCV y CV

3. Identificación de las variables por modelo (ver cuadro 1), a saber:

i) $\mathrm{MPH}$ : teorías primarias y secundarias, escala, incremento en el valor de la propiedad, modelo estadístico, distancia, criterio y particularidades.

ii) MCV: teorías primarias y secundarias, escala, valor de la visita, particularidades.

iii) VC: teorías primarias y secundarias, escala, modelo estadístico, criterio, valor encontrado, particularidades.

Cuadro 1. Variables de análisis

\begin{tabular}{|c|c|c|c|c|c|c|c|c|}
\hline & $\begin{array}{c}\text { Teorías } \\
\text { primarias } \\
\text { secundarias }\end{array}$ & Escala & $\begin{array}{c}\text { Incremento } \\
\text { en el valor } \\
\text { de la } \\
\text { propiedad }\end{array}$ & $\begin{array}{c}\text { Modelo } \\
\text { estadístico } \\
\text { adoptado }\end{array}$ & $\begin{array}{c}\text { Distancia } \\
\text { del } \\
\text { parque }\end{array}$ & $\begin{array}{c}\text { Resultado } \\
\text { del } \\
\text { criterio }\end{array}$ & $\begin{array}{c}\text { Valor } \\
\text { de la } \\
\text { visita }\end{array}$ & $\begin{array}{c}\text { Particularidades: } \\
\text { tipo de estudio, } \\
\text { divisa, región o } \\
\text { tiempo }\end{array}$ \\
\hline $\mathrm{MPH}$ & $\mathrm{X}$ & $\mathrm{X}$ & $\mathrm{X}$ & $\mathrm{X}$ & $\mathrm{X}$ & $\mathrm{X}$ & & $\mathrm{X}$ \\
\hline $\mathrm{MCV}$ & $\mathrm{X}$ & $\mathrm{X}$ & & & & & $\mathrm{X}$ & $\mathrm{X}$ \\
\hline $\mathrm{CV}$ & $\mathrm{X}$ & $\mathrm{X}$ & & $\mathrm{X}$ & & $\mathrm{X}$ & $\mathrm{X}$ & $\mathrm{X}$ \\
\hline
\end{tabular}

Fuente: elaboración propia 


\section{Resultados}

Los resultados se presentan en tres secciones de acuerdo a la categorización MPH, MCV, VC.

\section{A. Modelo de Precios Hedónicos (MPH)}

Se identifican tres teorías primarias (4.54\%); una teoría secundaria (1.51\%); un artículo de opinión (1.51\%); siete de revisión o metanálisis (10.60\%); un informe corporativo (1.51\%) y 53 estudios de caso (80.30\%). Las tres teorías primarias que fueron posibles de identificar confluyen desde diferentes enfoques y perspectivas. Lo que resalta es la idea de identificar la disposición a pagar por las características de un determinado bien -y no por el bien mismo- desde un enfoque que no está basado en el mercado. El MPH ha permanecido vigente por décadas con tan solo ligeras variantes en su espectro de aplicación y en su método, pero conservando su estructura esencial.

La excepción la marca el trabajo de Ferreira y Moro (2010), teoría secundaria que utiliza el MPH bajo el concepto bienestar subjetivo. Por otra parte, el artículo de opinión de Allen, Stevens y More (1985) presenta una discusión acerca del estatus del tema hasta ese tiempo, mientras que la revisión de Crompton (2005) la extiende 20 años más. En otros trabajos de revisión, Colwell y Dilmore (1999) hurgaron muy profundo para abrir el debate acerca de quién fue el primero en proponer el MPH. Se identificaron dos estudios acumulativos tipo metanálisis (Brander y Koetse, 2011; Perino et al., 2014); así como el trabajo de Crompton (2001), quien recapituló acerca de la evidencia empírica disponible hasta esa fecha. La revisión de la pertinencia de los modelos estadísticos, sus alcances y limitaciones, así como sus marcos de aplicación, se ha llevado a profundidad (Halvorsen y Pollakowski, 1981 elaboraron un informe corporativo contextualizando la revisión en los parques urbanos, resaltando los trabajos más relevantes. En el cuadro 2 se resumen los hallazgos de los grupos antes mencionados. Por último, el grupo más grande de artículos corresponde a la categoría estudio de caso, mismo que se analiza aparte.

En lo que respecta a los países de las publicaciones, Estados Unidos domina con 33 (50\%), seguido de Europa con 16 (24.24\%), mientras que Asia y Oceanía cuentan con 10 (15.15\%) y Latinoamérica con seis (9.09\%). De África destaca un trabajo (1.5\%), el de Abidoye y Chan (2017). Es de notarse que entre Estados Unidos y Europa suman el $75 \%$ de las publicaciones. Siguiendo a Abidoye y Chan (2017), los trabajos de África no se consideraron debido a que son cuestionables, tanto en su escala de aplicación, como en su instrumentación metódica, ya que se trata de un mercado altamente heterogéneo, cuya formalidad es todavía incipiente.

En los 53 estudios de caso que fue posible identificar (cuadro 3), Estados Unidos se posiciona en primer lugar con 24 publicaciones (45.28\%). Europa ocupa el segundo lugar con 14 (26.41\%), mientras que Asia y Oceanía en tercero con nueve (16.98\%). Por último, Latinoamérica cuenta con seis publicaciones (11.32\%). Tal como se especificó en el método, los estudios de caso se dividieron en las categorías: escala, modelo estadístico y resultados. En cuanto a la categoría escala, se clasificaron las publicaciones en cinco subcategorías de acuerdo con el nivel de cobertura del estudio: ciudad, condado, regional, zonal y parque. 
Cuadro 2. Publicaciones relativas a las categorías teoría primaria, teoría secundaria, opinión, revisiones e informes corporativos del $\mathrm{MPH}$.

\begin{tabular}{|c|c|c|}
\hline Fuente & Categoría & Comentario \\
\hline Lancaster (1966) & Teoría primaria & $\begin{array}{l}\text { Teoría de la Demanda de las Características: la utilidad } \\
\text { del consumidor está en función de las características de } \\
\text { un bien. }\end{array}$ \\
\hline Griliches (1971) & Teoría primaria & $\begin{array}{l}\text { Reflexiones sobre el estado del arte en ajustes de calidad, } \\
\text { cómo se percibe y cómo impacta el índice de precios. } \\
\text { Fuente frecuentemente citada. }\end{array}$ \\
\hline Rosen (1974) & Teoría primaria & $\begin{array}{l}\text { Cobra consistencia la teoría del MPH, se provee del } \\
\text { método de análisis. Es la fuente más reconocida y citada. }\end{array}$ \\
\hline $\begin{array}{l}\text { Ferreira y Moro } \\
(2010)\end{array}$ & Teoría secundaria & $\begin{array}{l}\text { El MPH da un giro hacia el bienestar subjetivo, además de } \\
\text { presentar alternativas para la violación del supuesto de } \\
\text { la condición de equilibrio. }\end{array}$ \\
\hline $\begin{array}{l}\text { Allen, Stevens y } \\
\text { More, (1985) }\end{array}$ & Opinión & $\begin{array}{l}\text { Crítica al modelo general, se analizan alternativas y se } \\
\text { advierte que el MPH subestima los beneficios de los } \\
\text { parques. }\end{array}$ \\
\hline $\begin{array}{l}\text { Halvorsen y } \\
\text { Pollakowski (1981) }\end{array}$ & $\begin{array}{l}\text { Revisión: modelo } \\
\text { estadístico }\end{array}$ & $\begin{array}{l}\text { No es posible especificar, en lo general y con bases } \\
\text { teóricas, la forma funcional de la ecuación para modelar } \\
\text { el esquema de precios hedónicos. }\end{array}$ \\
\hline $\begin{array}{l}\text { Cropper, Deck y } \\
\text { McConnell, (1988) }\end{array}$ & $\begin{array}{l}\text { Revisión: modelo } \\
\text { estadístico }\end{array}$ & $\begin{array}{l}\text { Los errores en la medición de los precios de los atributos } \\
\text { marginales varían de acuerdo con la función de precios } \\
\text { que se utilice. }\end{array}$ \\
\hline $\begin{array}{l}\text { Colwell y Dilmore } \\
\text { (1999) }\end{array}$ & Revisión: origen & $\begin{array}{l}\text { Examina el origen del MPH y especula acerca del creador } \\
\text { del concepto. }\end{array}$ \\
\hline Crompton (2001) & $\begin{array}{l}\text { Revisión: } \\
\text { evidencia } \\
\text { empírica }\end{array}$ & $\begin{array}{l}\text { Se confirma el principio de proximidad en } 25 \text { de } 30 \\
\text { estudios. Existe una mayor disposición a pagar por } \\
\text { viviendas cercanas a parques. }\end{array}$ \\
\hline Crompton (2005) & $\begin{array}{l}\text { Revisión: } \\
\text { evidencia } \\
\text { empírica }\end{array}$ & $\begin{array}{l}\text { Da continuidad al estudio anterior: encuentra que } 20 \% \\
\text { es una buena estimación para el incremento de precios } \\
\text { de viviendas cercanas a parques. }\end{array}$ \\
\hline $\begin{array}{l}\text { Brander y Koetse } \\
(2011)\end{array}$ & $\begin{array}{l}\text { Revisión: } \\
\text { metanálisis }\end{array}$ & $\begin{array}{l}\text { Análisis acumulativo de } 12 \text { estudios: el valor se relaciona } \\
\text { con la densidad poblacional, pero no con el ingreso. }\end{array}$ \\
\hline Perino et al., (2014) & $\begin{array}{l}\text { Revisión: } \\
\text { metanálisis }\end{array}$ & Considera solo estudios de caso en UK. \\
\hline $\begin{array}{l}\text { Harnik y Welle } \\
(2009)\end{array}$ & $\begin{array}{l}\text { Informe } \\
\text { corporativo }\end{array}$ & $\begin{array}{l}\text { Reporte de un coloquio de expertos enfocado en } \\
\text { valoración económica de parques urbanos. }\end{array}$ \\
\hline
\end{tabular}

Fuente: elaboración propia 
Cuadro 3. Estudios de caso adoptando el MPH para el periodo 1966-2020.

\begin{tabular}{|c|c|c|c|c|c|}
\hline \multirow[t]{2}{*}{ Fuente } & \multirow[t]{2}{*}{ País } & \multirow[t]{2}{*}{ Escala } & \multirow{2}{*}{$\begin{array}{c}\text { Modelo } \\
\text { estadístico }\end{array}$} & \multicolumn{2}{|c|}{ Resultado } \\
\hline & & & & Criterio & Valor \\
\hline $\begin{array}{l}\text { Araque, Gutiérrez y } \\
\text { Quenguan (2017) }\end{array}$ & Colombia & Zonal & 3 & $\begin{array}{c}\text { Dos } \\
\text { cuadras }\end{array}$ & $1: 0.23-1.18^{a}$ \\
\hline Barnett (1985) & Australia & Ciudad & 1,3 & $\begin{array}{c}\text { No } \\
\text { reportado }\end{array}$ & No estimado \\
\hline Bengochea (2003) & España & Ciudad & 1,3 & $100 \mathrm{~m}$ & $€ 1,800$ \\
\hline Biao et al., (2012) & China & Zonal & $1,2,3$ & $0.8-1.6 \mathrm{~km}$ & $0.5-14.1 \%$ \\
\hline $\begin{array}{l}\text { Bolitzer y Netusil } \\
(2000)\end{array}$ & $\begin{array}{l}\text { Estados } \\
\text { Unidos de } \\
\text { América }\end{array}$ & Ciudad & 1,2 & $1500 \mathrm{ft}$ & $\$ 1,2-\$ 2.1 \mathrm{~K}$ \\
\hline Chen y Jim (2010) & China & Ciudad & 2 & $450 \mathrm{~m}$ & $4.67 \%$ \\
\hline Cho et al., (2014) & $\begin{array}{l}\text { Estados } \\
\text { Unidos de } \\
\text { América }\end{array}$ & Condado & 3 & $\begin{array}{l}1 \text { minuto } \\
\text { en auto }\end{array}$ & $\$ 207 /$ acre \\
\hline $\begin{array}{l}\text { Cho, Kim y Roberts } \\
\text { (2011) }\end{array}$ & $\begin{array}{l}\text { Estados } \\
\text { Unidos de } \\
\text { América }\end{array}$ & Condado & 2 & 1 milla & $2.41 \%$ \\
\hline Cho et al., (2009)a & $\begin{array}{l}\text { Estados } \\
\text { Unidos de } \\
\text { América }\end{array}$ & Condado & 2 & $1000 \mathrm{ft}$ & \$1.4 K \\
\hline Cho et al., (2009)b & $\begin{array}{l}\text { Estados } \\
\text { Unidos de } \\
\text { América }\end{array}$ & Regional & 2 & n.p/d. & $\$ 950.00$ \\
\hline $\begin{array}{l}\text { Cho, Bowker y Park, } \\
(2006)\end{array}$ & $\begin{array}{c}\text { Estados } \\
\text { Unidos de } \\
\text { América } \\
\end{array}$ & Condado & 2 & $1000 \mathrm{ft}$ & $\$ 172.00$ \\
\hline Conway et al., ( 2010) & $\begin{array}{l}\text { Estados } \\
\text { Unidos de } \\
\text { América }\end{array}$ & Zonal & 2 & $300 \mathrm{ft}$ & $\$ 2,5 \mathrm{~K}$ \\
\hline $\begin{array}{l}\text { Correll, Lillydahl y } \\
\text { Singell (1978) }\end{array}$ & $\begin{array}{c}\text { Estados } \\
\text { Unidos de } \\
\text { América }\end{array}$ & Ciudad & 1 & $1000 \mathrm{ft}$ & $\$ 14.20 / \mathrm{ft}^{2}$ \\
\hline $\begin{array}{l}\text { Dehring y Dunse } \\
(2006)\end{array}$ & Reino Unido & Zonal & 2 & $800 \mathrm{~m}$ & $17.6 \%$ \\
\hline $\begin{array}{l}\text { Engström y Gren } \\
(2017)\end{array}$ & Suecia & Ciudad & 2 & $100 \mathrm{~m}$ & $1 \%$ \\
\hline $\begin{array}{l}\text { De Frutos y Esteban } \\
(2006)\end{array}$ & España & Ciudad & 2 & 15 minutos & $€ 238 /$ aก̃o \\
\hline Ferreira y Moro, 2010) & Reino Unido & Regional & 2 & $\begin{array}{c}\text { No } \\
\text { reportado }\end{array}$ & No estimado \\
\hline $\begin{array}{l}\text { Franco y Macdonald } \\
(2016)\end{array}$ & Portugal & Ciudad & 2 & $1 \mathrm{~km}^{2}$ & $€ 400$ \\
\hline Gruehn (2008) & Alemania & Regional & 6 & $500 \mathrm{~m}$ & $18.9 \%$ \\
\hline
\end{tabular}




\begin{tabular}{|c|c|c|c|c|c|}
\hline $\begin{array}{l}\text { Hagerty, Stevens y } \\
\text { More (1982) }\end{array}$ & $\begin{array}{l}\text { Estados } \\
\text { Unidos de } \\
\text { América }\end{array}$ & Ciudad & 2 & $2000 \mathrm{ft}$ & $\$ 19.8 \mathrm{~K} / \mathrm{acre}$ \\
\hline $\begin{array}{l}\text { Hammer, Coughlin y } \\
\text { Horn (1974) }\end{array}$ & $\begin{array}{l}\text { Estados } \\
\text { Unidos de } \\
\text { América }\end{array}$ & Parque & 1,3 & $1100 \mathrm{ft}$ & $9 \%$ \\
\hline Harnik y Welle (2009) & $\begin{array}{l}\text { Estados } \\
\text { Unidos de } \\
\text { América }\end{array}$ & Parque & 6 & $500 \mathrm{ft}$ & $5 \%$ \\
\hline $\begin{array}{l}\text { Hatton MacDonald et } \\
\text { al., (2010) }\end{array}$ & Australia & Ciudad & 2 & $\begin{array}{c}\text { No } \\
\text { reportado }\end{array}$ & Significativo \\
\hline Jansson y Axel (2000) & Chile & Ciudad & 5 & $\begin{array}{c}\text { No } \\
\text { reportado }\end{array}$ & Significativo \\
\hline Jim y Chen (2010) & Japón & Zonal & 2 & Vista & $16.88 \%$ \\
\hline $\begin{array}{l}\text { Kitchen y Hendon } \\
\text { (1967) }\end{array}$ & $\begin{array}{l}\text { Estados } \\
\text { Unidos de } \\
\text { América }\end{array}$ & Parque & 1 & $\begin{array}{c}\text { No } \\
\text { reportado }\end{array}$ & No estimado \\
\hline $\begin{array}{l}\text { Kong, Yin y Nakagoshi } \\
(2007)\end{array}$ & China & Ciudad & 1,2 & $300 \mathrm{~m}$ & $5 \%$ \\
\hline Kovacs (2012) & $\begin{array}{l}\text { Estados } \\
\text { Unidos de } \\
\text { América }\end{array}$ & Parque & 2 & $800 \mathrm{~m}$ & $20 \%$ \\
\hline $\begin{array}{l}\text { Liebelt, Bartke y } \\
\text { Schwarz (2018) }\end{array}$ & Alemania & Ciudad & 1 & $300 \mathrm{~m}$ & $€ 1.52 / \mathrm{m}^{2}$ \\
\hline Liu y Hite (2013) & $\begin{array}{c}\text { Estados } \\
\text { Unidos de } \\
\text { América }\end{array}$ & Ciudad & 2 & $\begin{array}{c}\text { No } \\
\text { reportado }\end{array}$ & No estimado \\
\hline Luttik (2000) & Países Bajos & Regional & 1 & Vista & $7-8 \%$ \\
\hline $\begin{array}{l}\text { Lutzenhiser y Netusil } \\
\text { (2001) }\end{array}$ & $\begin{array}{c}\text { Estados } \\
\text { Unidos de } \\
\text { América }\end{array}$ & Condado & 1,5 & $1500 \mathrm{ft}$ & $\$ 1.2 \mathrm{~K}$ \\
\hline Mayor et al. (2009) & Irlanda & Ciudad & 2 & $2,000 \mathrm{~m}$ & $9-11 \%$ \\
\hline McLeod (1984) & Australia & Ciudad & 4 & 2.7 calles & $3 \%$ \\
\hline Morales (1980) & $\begin{array}{l}\text { Estados } \\
\text { Unidos de } \\
\text { América }\end{array}$ & Ciudad & 6 & $\begin{array}{c}\text { No } \\
\text { reportado }\end{array}$ & $6 \%$ \\
\hline $\begin{array}{l}\text { More, Stevens y Allen } \\
(1988)\end{array}$ & $\begin{array}{l}\text { Estados } \\
\text { Unidos de } \\
\text { América }\end{array}$ & Zonal & 2 & $2,000 \mathrm{ft}$ & $\$ 2.6 \mathrm{~K}$ \\
\hline $\begin{array}{l}\text { Moreno y Alvarado } \\
(2011)\end{array}$ & México & Zonal & 2,3 & $\begin{array}{c}\text { No } \\
\text { reportado }\end{array}$ & $0.05 \% / \mathrm{m}$ \\
\hline $\begin{array}{l}\text { Noor, Asmawi y } \\
\text { Abdullah (2015) }\end{array}$ & Malasia & Ciudad & 1 & $400 \mathrm{~m}$ & $3-12 \%$ \\
\hline Panduro y Veie (2013) & Dinamarca & Ciudad & 6 & $100 \mathrm{~m}$ & $3 \%$ \\
\hline Penagos (2005) & Colombia & Ciudad & 5 & $200 \mathrm{~m}$ & $\$ 4.8 \mathrm{~K}$ \\
\hline
\end{tabular}




\begin{tabular}{|c|c|c|c|c|c|}
\hline $\begin{array}{l}\text { Poudyal, Hodges y } \\
\text { Merrett (2009) }\end{array}$ & $\begin{array}{c}\text { Estados } \\
\text { Unidos de } \\
\text { América }\end{array}$ & Ciudad & 2 & Tamaño & $\$ 160.00$ \\
\hline $\begin{array}{l}\text { Romero y Vargas } \\
(2016)\end{array}$ & Colombia & Zonal & 1,3 & Área & $2.24 \% / \mathrm{m}^{2}$ \\
\hline Sander y Haight (2012) & $\begin{array}{l}\text { Estados } \\
\text { Unidos de } \\
\text { América }\end{array}$ & Ciudad & 2 & $500 \mathrm{~m}$ & $\$ 129-\$ 216$ \\
\hline $\begin{array}{l}\text { Sander, Polasky y } \\
\text { Haight (2010) }\end{array}$ & $\begin{array}{l}\text { Estados } \\
\text { Unidos de } \\
\text { América }\end{array}$ & Regional & 2 & $100-250 \mathrm{~m}$ & $\$ 0.836-1.3 \mathrm{~K}$ \\
\hline $\begin{array}{l}\text { Sander y Polasky } \\
(2009)\end{array}$ & $\begin{array}{l}\text { Estados } \\
\text { Unidos de } \\
\text { América }\end{array}$ & Condado & 2 & $500 \mathrm{~m}$ & $\$ 136.00$ \\
\hline Saphores y Li (2012) & $\begin{array}{l}\text { Estados } \\
\text { Unidos de } \\
\text { América }\end{array}$ & Ciudad & 2,5 & $500 \mathrm{~m}$ & Significativo \\
\hline Schläpfer et al. (2015) & Suecia & Zonal & 2 & $300-1500 \mathrm{~m}$ & No concluyente \\
\hline Tajima (2003) & $\begin{array}{l}\text { Estados } \\
\text { Unidos de } \\
\text { América }\end{array}$ & Zonal & 2 & $300 \mathrm{~m}$ & $\$ 190-\$ 210$ \\
\hline Troy y Grove (2008) & $\begin{array}{c}\text { Estados } \\
\text { Unidos de } \\
\text { América }\end{array}$ & Ciudad & $2,3,5$ & $450 \mathrm{~m}$ & $\$ 1,800$ \\
\hline Tyrväinen (1997) & Finlandia & Ciudad & 1,2 & $200 \mathrm{~m}$ & No concluyente \\
\hline $\begin{array}{l}\text { Tyrväinen y Miettinen } \\
(2000)\end{array}$ & Finlandia & Ciudad & 5 & $400 \mathrm{~m}$ & $4.9 \%$ \\
\hline $\begin{array}{l}\text { Vargas y Roldán } \\
(2017)\end{array}$ & Colombia & Ciudad & 2 & $332 \mathrm{~m}$ & $\$ 139 / 100 \mathrm{~m}$ \\
\hline $\begin{array}{l}\text { Weigher y Zerbst } \\
\text { (1973) }\end{array}$ & China & Ciudad & 2 & $\begin{array}{c}\text { No } \\
\text { reportado }\end{array}$ & 7,897 Yuan \\
\hline
\end{tabular}

${ }^{a}$ Proporción de valor, esto es, una vivienda cercana al parque aumenta su valor en una proporción de 0.23-1.18 en relación con una lejana al parque. Fuente: elaboración propia

La división política condado solo se presenta en los Estados Unidos, así que se da cuenta del número de estudios a este nivel, pero después se agrupan en la categoría ciudad para mejorar el grado de comparabilidad con otras regiones. En la categoría modelo estadístico se identificó en cada publicación el formato del modelo estadístico de regresión que se utilizó, aclarando que en muchos casos se utilizó más de un formato. Las subcategorías del modelo son: lineal (1), semilogarítmico (2), logarítmico (3), no lineal (4), Box-Cox (5) y no reportado (6). 
A nivel ciudad se encontraron 28 publicaciones (52.83\%), mientras que a nivel condado seis (11.32\%); si se agregan estas dos subcategorías se tienen 34 publicaciones relativas a una ciudad y sus alrededores (64.15\%). En segundo lugar, están las publicaciones a nivel zonal, es decir, aquellas que consideran solo un sector de una ciudad, con 10 publicaciones (18.86\%). Las publicaciones a nivel de un parque en específico son seis (11.32\%), a la vez que los trabajos a nivel regional, es decir, considerando más de una ciudad, son cinco $(9.43 \%)$.

Otra categoría de interés del MPH es el tipo de modelo estadístico que se ha adoptado para conducir los estudios. En muchas investigaciones, se reportan problemas con la validez del modelo de regresión, principalmente por la presencia de multicolinealidad y heterocedasticidad. Es frecuente que algunas variables estructurales de las viviendas estén relacionadas directamente. Por ejemplo, es de esperarse que lotes más grandes cuenten con un mayor número de habitaciones, lo que hace que la validez del modelo se vea comprometida. Por otra parte, la normalidad e independencia del error aleatorio no siempre está presente. Es común que los residuales del modelo estén en función de una o más variables, lo que también afecta la validez del modelo. Para remediar esta situación se han propuesto esquemas tales como la transformación Box-Cox, además de las modalidades logarítmicas, semilogarítmicas o no lineales.

El modelo estadístico más utilizado es el semilogarítmico. Este formato normalmente se utiliza en conjunto con el lineal, siendo este último usualmente la primera opción, que se descarta si la validez se ve comprometida. Recuérdese que la modelación econométrica no está exenta de errores y que es hasta cierto punto limitada. Para que el MPH sea válido es necesario suponer: (1) que el ámbito espacial urbano estudiado (ciudad, condado, entre otros) se puede considerar como un solo mercado; (2) que el mercado de la vivienda en ese ámbito está cerca del equilibrio; y (3) que el modelo estadístico de regresión utilizado es adecuado (Cropper, Deck y McConnell, 1988). En este último punto, es donde resalta la importancia de hacer notar que el modelo que ha resultado más pertinente es el semilogarítmico. Por otra parte, tan solo un estudio reporta un modelo no lineal, otros cinco utilizaron una transformación Box-Cox y en cuatro casos no se reportó en la publicación el tipo de modelo utilizado.

Dentro de la categoría resultados se analizan dos subcategorías: criterio y valor. El criterio se refiere al potencial de cobertura del parque urbano, es decir, hasta dónde el consumidor está dispuesto a pagar y cuáles son las fronteras de esta disposición. La gran mayoría de las publicaciones considera la distancia al parque como una de las variables de localización. Los resultados que arrojan las publicaciones muestran que el signo del coeficiente de esta variable es negativo, lo que significa que conforme una vivienda está más alejada del parque, la disposición a pagar disminuye hasta que el efecto de la cercanía desaparece. Las cifras encontradas en las publicaciones revisadas promedian ponderadamente una distancia de $520.22 \mathrm{~m}$. Para Estados Unidos se encontró una distancia promedio de $491.31 \mathrm{~m}$, mientras que para Europa el promedio es de $581.81 \mathrm{~m}$. En región de Asia y Oceanía la distancia promedio de influencia del parque llega hasta los $650 \mathrm{~m}$; a su vez, en Latinoamérica el promedio es de $310 \mathrm{~m}$.

Por último, el objetivo del MPH es estimar el VNM, es decir, la disposición a pagar por contar con los servicios de un parque. Los resultados encontrados en esta subcategoría muestran que el valor reportado en las publicaciones no sigue un formato estándar. La forma más frecuente de dar cuenta del valor del parque es como porcentaje del precio de las viviendas (ver cuadro 4). Otras 
formas incluyen el valor del parque expresado como un radio de los costos de mantenimiento del parque, es decir, la cantidad de valor que brinda el espacio por cada unidad de numerario que se invierte en su mantenimiento y conservación. También se expresa como una cantidad fija de aumento en el precio de la vivienda, lo que implica una estimación del valor de las viviendas del vecindario y una estimación incremental. Asimismo, como una razón de cambio en el precio al alejarse del parque o al aumentar en una unidad de superficie el tamaño de este, lo que se deriva del signo y coeficiente de la variable de localización correspondiente al parque en el modelo.

Cuadro 4. Porcentaje de aumento en el precio de las viviendas por la presencia del parque.

\begin{tabular}{|c|c|c|c|}
\hline Región & Mínimo & Máximo & Promedio \\
\hline Estados Unidos & 2.41 & 20 & 5.60 \\
\hline Europa & 1 & 18.9 & 12.68 \\
\hline Asia y Oceanía & 3 & 16.88 & 10.53 \\
\hline Latinoamérica & \multicolumn{3}{|c|}{ No reportado } \\
\hline
\end{tabular}

Fuente: elaboración propia

\section{B. Modelo del costo del viaje (MCV)}

Los resultados encontrados en la revisión de las publicaciones del MCV para la valoración de parques urbanos y periurbanos se presentan a continuación. Fue posible identificar 14 publicaciones, el periodo comprende 52 años (1966-2018), con una referencia al trabajo seminal de Hotelling (1949). Se identificaron tres publicaciones consideradas pioneras en el tema $(21.42 \%)$ y 11 estudios de caso (78.71\%). No fue posible identificar teorías secundarias ni reportes corporativos. Las teorías primarias se enlistan en la tabla 5.

Cuadro. 5. Publicaciones de teorías primarias del MCV.

\begin{tabular}{|l|l|l|}
\hline Fuente & Categoría & Comentario \\
\hline Cesario (1976) & $\begin{array}{l}\text { Teoría } \\
\text { primaria }\end{array}$ & $\begin{array}{l}\text { Establece la teoría y el método de estudio. (Modelo de } \\
\text { gravedad) }\end{array}$ \\
\hline $\begin{array}{l}\text { Clawson y Knetsch } \\
(2013)\end{array}$ & $\begin{array}{l}\text { Teoría } \\
\text { primaria }\end{array}$ & $\begin{array}{l}\text { Exceso del consumidor basada en oportunidades: valor } \\
\text { no basado en el mercado de la recreación }\end{array}$ \\
\hline Hotelling (1949) & $\begin{array}{l}\text { Teoría } \\
\text { primaria }\end{array}$ & Inicia la teoría del valor de la recreación \\
\hline
\end{tabular}

Fuente: elaboración propia 
Los 11 estudios de caso identificados se presentan en la tabla 6. De acuerdo con las regiones, se tiene una publicación de Estados Unidos (9.09\%), tres de Europa (27.27 \%), dos de Asia y Oceanía (18.18\%), y cinco de Latinoamérica (45.45\%). En cuanto a la escala, predominan los estudios en parques periurbanos con cuatro (36.36\%), seguidos de los estudios zonales (más de un parque en una parte de una ciudad) con tres (27.27\%), dos a nivel unidad o parque (18.18\%), un estudio a nivel regional (más de una ciudad) $(9.09 \%$ ) y uno a nivel país $(9.09 \%)$

Cuadro 6. Estudios de caso adoptando el MCV.

\begin{tabular}{|c|c|c|c|c|}
\hline Fuente & País & Escala & $\begin{array}{c}\text { Valor de la } \\
\text { visita }\end{array}$ & Comentario \\
\hline $\begin{array}{l}\text { Bartczak et al., } \\
(2008)\end{array}$ & Polonia & País & $€ 0.64-6.93$ & $\begin{array}{l}\text { Encuentra que los espacios } \\
\text { verdes urbanos de Polonia } \\
\text { están subvaluados. }\end{array}$ \\
\hline $\begin{array}{l}\text { Chaudhry y Tewari } \\
(2006)\end{array}$ & India & $\begin{array}{c}\text { Regional } \\
\text { periurbana }\end{array}$ & Rs\$308.00 & Compara con VC. \\
\hline $\begin{array}{l}\text { Escobar y Erazo } \\
(2006)\end{array}$ & Colombia & $\begin{array}{c}\text { Parque } \\
\text { periurbano }\end{array}$ & $C \$ 4,395.00$ & Compara con VC. \\
\hline $\begin{array}{l}\text { Escobar y Ramírez, } \\
(2009)\end{array}$ & Colombia & $\begin{array}{c}\text { Parque } \\
\text { periurbano }\end{array}$ & US\$350.00 & Compara con VC. \\
\hline Gándara (2006) & México & Parque urbano & $\begin{array}{c}M \times \$ 42.15- \\
64.54\end{array}$ & $\begin{array}{l}\text { Brinda pautas para una } \\
\text { mejor gestión del espacio } \\
\text { estudiado. }\end{array}$ \\
\hline $\begin{array}{l}\text { Hanauer y Reid } \\
\text { (2017) }\end{array}$ & $\begin{array}{l}\text { Estados } \\
\text { Unidos de } \\
\text { América }\end{array}$ & $\begin{array}{l}\text { Parque } \\
\text { periurbano }\end{array}$ & US\$13.70 & $\begin{array}{l}\text { El MCV subestima el valor } \\
\text { real del espacio. }\end{array}$ \\
\hline $\begin{array}{l}\text { Lockwood y Tracy } \\
\text { (1995) }\end{array}$ & Australia & Parque urbano & AU\$25.81 & $\begin{array}{l}\text { Beneficios entre: AU\$23-33 } \\
\text { millones }\end{array}$ \\
\hline $\begin{array}{l}\text { Martínez-Cruz } \\
(2005)\end{array}$ & México & $\begin{array}{c}\text { Parque } \\
\text { periurbano }\end{array}$ & $\begin{array}{c}M X \$ 66.3- \\
115.5 \mathrm{M}\end{array}$ & $\begin{array}{l}\text { Utilizó estratificación } \\
\text { endógena. }\end{array}$ \\
\hline $\begin{array}{l}\text { Martínez-Cruz y } \\
\text { Sainz (2017) }\end{array}$ & México & Zonal periurbana & $\begin{array}{l}\text { US\$12-33 } \\
\text { US\$2-6 }\end{array}$ & $\begin{array}{l}\text { US\$223 anuales, US\$158 } \\
\text { anuales }\end{array}$ \\
\hline $\begin{array}{l}\text { Willis y Benson } \\
(1989)\end{array}$ & Reino Unido & Zonal periurbana & $£ 1.90$ & $\begin{array}{l}\text { Beneficios entre: } £ 14-45 \\
\text { millones }\end{array}$ \\
\hline $\begin{array}{l}\text { Willis y Garrod } \\
(1991)\end{array}$ & Reino Unido & Zonal periurbana & $\begin{array}{l}£ 0.40- \\
£ 2.32\end{array}$ & $\begin{array}{l}\text { Beneficios de } £ 3.35 \\
\text { millones }\end{array}$ \\
\hline
\end{tabular}

Fuente: elaboración propia

En todos los estudios de caso se reportaron valores del costo del viaje positivos, es decir, las personas perciben una utilidad al visitarlos. Lo anterior significa que los beneficios percibidos al utilizar el parque, son mayores que los desembolsos hechos en el traslado, la entrada al lugar y el costo de oportunidad por el tiempo dedicado a la visita. Destacan los estudios de caso en el Reino Unido, Australia y México, con cifras de valoración millonarias, considerando la estimación 
del número de visitantes totales. El formato de reporte del valor de la visita es similar entre las publicaciones consideradas. La baja cantidad de publicaciones encontradas en esta categoría no permite un cálculo de valor por región.

\section{c. Valoración contingente (VC)}

Por otra parte, entre las 40 publicaciones relativas a la VC, se identificó una teoría primaria, un artículo de opinión, cuatro revisiones y 34 estudios de caso. El cuadro 7 lista las publicaciones correspondientes a las categorías teoría primaria, opinión y revisión. Las publicaciones correspondientes a la categoría estudio de caso se analizan por separado.

Cuadro. 7. Publicaciones de la VC correspondientes a las categorías teoría primaria, opinión y revisión.

\begin{tabular}{|l|l|l|}
\hline \multicolumn{1}{|c|}{ Fuente } & \multicolumn{1}{c|}{ Categoría } & \multicolumn{1}{c|}{ Comentario } \\
\hline Davis (1963) & Teoría primaria & Marca el inicio de la VC. Trabajo multicitado. \\
\hline $\begin{array}{l}\text { Carson, Flores y Meade, } \\
(2001)\end{array}$ & Opinión & $\begin{array}{l}\text { Discute los temas principales del debate sobre la VC y } \\
\text { su validez en el uso pasivo. }\end{array}$ \\
\hline Arrow et al., (1993) & $\begin{array}{l}\text { Revisión: informe } \\
\text { oficial }\end{array}$ & $\begin{array}{l}\text { Informe de un panel de la NOAA de Estados Unidos. } \\
\text { Discute los formatos de entrevista. Frecuentemente } \\
\text { citado. }\end{array}$ \\
\hline $\begin{array}{l}\text { Borzykowski, Baranzini, } \\
\text { y Maradan (2018) }\end{array}$ & $\begin{array}{l}\text { Revisión: modelo } \\
\text { estadístico }\end{array}$ & $\begin{array}{l}\text { Discute los supuestos de la distribución de } \\
\text { probabilidad de la disposición a pagar. }\end{array}$ \\
\hline Carson et al. (1996) & $\begin{array}{l}\text { Revisión: } \\
\text { metanálisis }\end{array}$ & $\begin{array}{l}\text { Análisis acumulativo de 83 estudios: encuentra que las } \\
\text { estimaciones de VC son ligeramente menores que la } \\
\text { disposición a pagar. }\end{array}$ \\
\hline Thayer (1981) & $\begin{array}{l}\text { Revisión: } \\
\text { evidencia }\end{array}$ & $\begin{array}{l}\text { Revisa tres aspectos: naturaleza hipotética de la } \\
\text { encuesta, cuestionario y el proceso de subasta. }\end{array}$ \\
\hline
\end{tabular}

${ }^{a}$ National Oceanic and Atmospheric Administration. Fuente: elaboración propia.

Se aprecia que en Europa se originaron 13 trabajos (32.5\%), de Asia y Oceanía proceden ocho publicaciones (20\%), 10 de Latinoamérica (25\%), mientras que seis (15\%) son de Estados Unidos y tres (7.5\%) de África. En relación con los 34 estudios de caso que se identificaron (véase cuadro 8), 13 publicaciones son europeas (38.24\%); ocho de Asia y Oceanía (23.53\%) como de Latinoamérica provienen nueve (26.47\%); en tanto que tres son de África $(8.82 \%)$ y una de Estados Unidos (2.94\%).

El modelo estadístico de regresión logística Logit es el que más se ha utilizado, se identificaron 18 trabajos que lo adoptaron (más uno compartido). Por su parte el esquema Tobit se utilizó en tres estudios de caso (más uno compartido), a la vez que el Probit en uno. Dos estudios de caso construyeron ecuaciones estructurales para el análisis. En tres trabajos se utilizó un modelo lineal, sin que se especificara el tipo que se adoptó, mientras que en siete publicaciones no se reportó el tipo de modelo utilizado. 
Las valoraciones se condujeron con diferentes propósitos en mente. La principal motivación fue investigar la disposición a pagar por la preservación del espacio y de ahí surgen motivaciones muy variadas, que van desde descubrir qué tanto está presente en las personas la importancia del espacio y en cuánto lo valoran, hasta conocer la factibilidad económica de sustituir el uso de suelo de un espacio para convertirlo en parque. También se aprecian otras motivaciones, tales como la disposición a pagar por reducir el ruido en el parque, conocer el valor otorgado al espacio en un cierto periodo, así como comparar el resultado con el obtenido con el MCV.

Cuadro 8. Estudios de caso adoptando la VC el periodo 1963-2020.

\begin{tabular}{|c|c|c|c|c|c|}
\hline \multirow[t]{2}{*}{ Fuente } & \multirow[t]{2}{*}{ País } & \multirow[t]{2}{*}{ Escala } & \multirow{2}{*}{$\begin{array}{l}\text { Modelo } \\
\text { estadístico }\end{array}$} & \multicolumn{2}{|c|}{ Resultado } \\
\hline & & & & Comentario & Valor \\
\hline Calleja et al., (2017) & España & Parque & Logit & Ruido en parque & $€ 4.13-7.08$ \\
\hline Chaudhry y Tewari (2006) & India & Ciudad & $\mathrm{N} / \mathrm{R}$ & Compara con MCV & $\mathrm{RS} \$ 6.73$ \\
\hline Chen y Hua (2015) & Japón & Ciudad & Logit & Árboles urbanos & No reportado \\
\hline Chen y Jim (2012) & Japón & Regional & Logit & $\begin{array}{c}\text { Parques } \\
\text { periurbanos }\end{array}$ & HK\$101.00 \\
\hline Chen y Jim (2008) & China & Ciudad & Logit & Espacios verdes & RMB161.84 \\
\hline Chen y Qi (2018) & China & Parque & Logit & Bosque urbano & RMB13.79 \\
\hline Cook et al., (2018) & Islandia & Parque & Logit & Preservación & ISK24,790 \\
\hline $\begin{array}{l}\text { Del Saz-Salazar y Rausell- } \\
\text { Köster (2008) }\end{array}$ & España & Parque & Tobit & Ciclo de vida & $€ 61.7$ millones \\
\hline $\begin{array}{l}\text { Del Saz-Salazar y García } \\
\text { Menéndez, (2007) }\end{array}$ & España & Parque & Logit & Sustitución de uso & $€ 63.1$ millones \\
\hline Dumenu (2013) & Ghana & Parque & $\mathrm{N} / \mathrm{R}$ & Valor US\$15-31 & $\$ 2.7$ millones \\
\hline Escobar y Erazo (2006) & Colombia & Parque & Logit & Compara con MCV & $C \$ 2,000-6,000$ \\
\hline Escobar y Ramírez (2009) & Colombia & Parque & Logit & Disposición a pagar & US\$1,000.00 \\
\hline $\begin{array}{l}\text { Flores-Xolocotzi, González- } \\
\text { Guillén y de los Santos- } \\
\text { Posadas (2010) }\end{array}$ & México & Parque & Tobit & $\mathrm{MX} \$ 34.5 / \mathrm{Ha}$ & MX\$543.00 \\
\hline De Frutos y Esteban (2009) & España & Ciudad & Logit & $€ 2 / \mathrm{Ha}$ & $€ 47.00$ \\
\hline Hernández et al., (2013) & México & Parque & $\mathrm{N} / \mathrm{R}$ & Parque periurbano & $M X \$ 10-20$ \\
\hline Jim y Chen (2006) & China & Ciudad & Probit & RMB547 millones & $\begin{array}{l}\text { RMB17.40/p/ } \\
\mathrm{m}\end{array}$ \\
\hline Larqué et al. (2004) & México & Parque & Lineal & $\mathrm{MX} \$ 36.8 \mathrm{M}$ & $M X \$ 272.00$ \\
\hline $\begin{array}{l}\text { Latinopoulos, Mallios, y } \\
\text { Latinopoulos (2016) }\end{array}$ & Grecia & Parque & Logit & $\begin{array}{c}\text { Dos tipo de } \\
\text { respuesta }\end{array}$ & $€ 3.98-7.85$ \\
\hline Lo y Jim (2010) & Japón & Ciudad & Lineal & HK 46,458/5 años & HK77.43 \\
\hline $\begin{array}{l}\text { López-Mosquera, García y } \\
\text { Barrena (2014) }\end{array}$ & España & Parque & Estructural & Obligación moral & $€ 12.67$ \\
\hline $\begin{array}{l}\text { López-Mosquera y } \\
\text { Sánchez (2012) }\end{array}$ & España & Parque & Estructural & Obligación Moral & $€ 1.15$ \\
\hline $\begin{array}{l}\text { López-Mosquera y } \\
\text { Sánchez (2011) }\end{array}$ & España & Parque & Logit & Valores personales & $€ 1.33$ \\
\hline Majumdar et al., (2011) & $\begin{array}{l}\text { Estados } \\
\text { Unidos de } \\
\text { América }\end{array}$ & Ciudad & Logit & US\$81-167 M & US\$7.34-15.16 \\
\hline
\end{tabular}




\begin{tabular}{|c|c|c|c|c|c|}
\hline Muñoz et al. (2020) & México & Ciudad & $\mathrm{N} / \mathrm{R}$ & Disposición a pagar & US\$99.00 \\
\hline $\begin{array}{l}\text { Nielsen, Olsen y Lundhede } \\
\text { (2007) }\end{array}$ & Dinamarca & País & Logit & Arbolado urbano & DKK1,939.00 \\
\hline Neckel et al. (2020) & Brasil & Parque & $\mathrm{N} / \mathrm{R}$ & Disposición a pagar & $\mathrm{R} \$ 959,024.00$ \\
\hline Platania y Rizzo (2018) & Italia & Parque & Logit & Precio de entrada & $€ 2.00-5.00$ \\
\hline Rivas y Ramoni (2002) & Venezuela & Parque & Logit & Recuperación & $\begin{array}{c}\text { No } \\
\text { significativo }\end{array}$ \\
\hline Sepúlveda (2008) & Colombia & Parque & Logit & $\begin{array}{c}\text { Parque } \\
\text { metropolitano }\end{array}$ & $\mathrm{C} \$ 6,100 / \mathrm{mes}$ \\
\hline $\begin{array}{l}\text { Sirina, Hua y Gobert } \\
\text { (2017) }\end{array}$ & Francia & Parque & Logit, Tobit & Suburbano & $\begin{array}{c}\text { No } \\
\text { significativo }\end{array}$ \\
\hline $\begin{array}{l}\text { Tameko, Donfouet y Sikod } \\
\text { (2011) }\end{array}$ & Camerún & Parque & Logit & Preservación & CFA370.35 \\
\hline $\begin{array}{l}\text { Tibesigwa, Herbert y } \\
\text { Lokina (2020) }\end{array}$ & Tanzania & Parque & $\mathrm{N} / \mathrm{R}$ & Disposicion a pagar & $\begin{array}{l}\text { US\$0.10 - } \\
\text { US\$0.47 }\end{array}$ \\
\hline $\begin{array}{l}\text { Tyrväinen y Väänänen } \\
\text { (1998) }\end{array}$ & Finlandia & Ciudad & $\begin{array}{c}\text { Lineal, } \\
\text { Tobit }\end{array}$ & Preservación & $\begin{array}{l}\mathrm{F} \$ 0.82- \\
\text { 3.8/año }\end{array}$ \\
\hline Xu et al. (2020) & China & Ciudad & $\mathrm{N} / \mathrm{R}$ & Disposición a pagar & CNY 26.25 \\
\hline
\end{tabular}

Los formatos para reportar el valor encontrado en los estudios también tienen variaciones, predominando la forma disposición a pagar por persona, misma que al multiplicarse por el número de usuarios (demanda) en un determinado periodo, determina una estimación del valor del espacio en dicho periodo. También, dependiendo del formato que se haya establecido para el modelo econométrico, es posible ver los resultados expresados como valor por unidad de superficie.

\section{Discusión}

El esquema de valoración que más se ha adoptado es el MPH, particularmente en las últimas décadas, periodo en el que se ha incrementado notablemente el número de aplicaciones. En este sentido, Estados Unidos y Europa se destacan con el mayor número de investigaciones, principalmente a nivel ciudad. Una de las dificultades del MPH es identificar el efecto del parque en el precio de las viviendas ante un cúmulo de variables que también se asocian a su valor de mercado. Es de notarse que mientras mayor sea la heterogeneidad del tipo de vecindarios (intra-heterogeneidad) en las inmediaciones del parque y mientras más heterogéneo sea el tipo de vivienda (inter-heterogeneidad), más complicada es la modelación econométrica y mayor la dificultad de identificar el efecto del parque en el precio de la vivienda (Tyrväinen, 1997). El nivel de heterogeneidad del mercado de la vivienda explica, en parte, por qué los estudios son abundantes en países desarrollados, menos abundantes en países en desarrollo y escasos en otras regiones menos desarrollados.

Por otra parte, la validez del MPH depende en gran medida de la condición de equilibro de mercado y de la disponibilidad de datos, condiciones ambas muy difíciles de estar presentes 
en países en vías de desarrollo. En estos países, influyen en los mercados factores especulativos, malas prácticas gubernamentales y existe poca o nula disponibilidad de datos oficiales confiables. En contraste, en los países desarrollados las prácticas de mercado son mejores y existen datos confiables de censos y catastro. En Estados Unidos, así como en una gran parte de Europa, no presenta mayor dificultad obtener datos relativos a las características de las viviendas, así como el detalle de las transacciones de compra-venta de las mismas. Con estas circunstancias tan favorables, no es extraño que predominen los estudios económicos a una escala relativamente grande, como es la ciudad, inclusive a nivel condado, que engloba más de una ciudad.

La complejidad en la modelación econométrica que adopta el MPH se evidencia en el uso mayoritario de los modelos semilogarítmico y logarítmico como alternativas al modelo lineal simple. La incorporación de los atributos estructurales de las viviendas y de localización a un modelo econométrico puede traer consigo mucha dependencia implícita entre ellos. Lo anterior trae consigo multicolinealidad entre variables y hace necesario conferir aditividad al modelo, transformado a expresiones lineales las interacciones complejas entre estos atributos (Cropper, Deck y McConnell., 1988). La aditividad se consigue a través del uso de transformaciones logarítmicas que además subsanan la multicolinealidad y la heterocedasticidad, mejorando el comportamiento del error aleatorio del modelo (Halvorsen y Pollakowski, 1981). El reporte de los resultados del MPH no sigue un formato estándar. La distancia a la que el parque deja de tener influencia sobre el precio de las viviendas es más grande en la región de Asia y Oceanía, seguido de Europa y Estados Unidos, mientras que Latinoamérica es aún incierta por ser muy escaso el número de estudios hasta la fecha. Estos resultados parecen sugerir que la influencia del parque sobre el precio de las viviendas pudiera ser mayor en las regiones donde las ciudades son más compactas, especialmente en China y en menor grado en Europa (Dehring y Dunse, 2006). En relación con el promedio de aumento en el precio de las viviendas, se aprecia que en Europa alcanza el valor más alto, seguido de Asia y Oceanía, superando los dos dígitos, Estas dos regiones se caracterizan por ciudades muy compactas y con gran tradición por la apreciación de los parques urbanos y por el espacio público en general. En Estados Unidos este porcentaje se sitúa en 5.6\%, una cifra alrededor de la cual se ubica la disposición a pagar por contar con un parque. No fue posible identificar un porcentaje para Latinoamérica. El promedio global de aumento del precio de una vivienda por la presencia de un parque es de $9.68 \%$, con una distancia global promedio de $520.22 \mathrm{~m}$.

En lo que se refiere al MCV el número de estudios de caso es mucho menor y en su mayoría se refieren a parques periurbanos localizados en los linderos de una ciudad y no propiamente a parques urbanos. A pesar de que en los países desarrollados este modelo es ampliamente utilizado para la valoración de parques nacionales, lagos y playas (Hanauer y Reid, 2017), prácticamente no se adopta para la valoración del uso de parques urbanos. Solo fue posible identificar un estudio en Estados Unidos, dos en Europa y uno en Australia. Se puede especular que quizás no se perciba la necesidad de un ejercicio de valoración de un parque urbano mediante el MCV porque el valor de estos espacios sería algo que pudiera considerarse obvio. Después de todo existe en estos países una cultura de voluntarismo y participación ciudadana, además de una gran tradición por la apreciación de los parques. En lo que toca a Latinoamérica, el MCV tienen más pertinencia, ya que el costo del traslado, aun dentro de la misma ciudad, pudiera representar una proporción 
importante del ingreso diario de un trabajador promedio. Los resultados que arrojan los estudios de caso del MCV siguen un formato estándar porque precisamente el objetivo del modelo es calcular el valor del espacio con base en la estimación del valor de la visita. De esta manera, si se multiplica el valor de la visita por el número de visitantes en un cierto periodo, se obtiene el valor de uso del parque en ese periodo.

En lo que respecta a la VC, se encontró solo un estudio de caso en Estados Unidos, quizás por las mismas razones que se esgrimieron en la discusión del MVC. No obstante, en Asia y Oceanía, Europa, Latinoamérica y África si fue posible identificar algunos estudios de caso. Es claro en los resultados de los estudios de caso reportados que la VC se adopta tanto para valorar el uso como el no uso de un espacio. En unos casos se utiliza para exponer el valor del parque y así promover su preservación. En otros, se apela a la conciencia de las personas para que valoren el espacio por su importancia ambiental o de recreación; mientras que otros se buscan propósitos diversos, tales como estimar el valor a lo largo del ciclo de vida o el cambio de uso de suelo. Con excepción de dos estudios, y uno en donde no se reportó, en todos los demás se encontró una disposición a pagar positiva. La VC es muy versátil para utilizarse a nivel parque, pero conlleva la dificultad de las respuestas negativas y de protesta, por lo que su adopción debe prever estas dificultades (Cristeche y Penna, 2008).

\section{Conclusiones}

El número de estudios de valoración económica de los servicios que prestan los parques urbanos se ha incrementado en los últimas dos décadas. Casi tres de cada cuatro publicaciones se originan en Estados Unidos o Europa, regiones donde existe una gran tradición por la apreciación de los parques urbanos. Se nota una marcada prevalencia de publicaciones del tipo estudio de caso, lo que sugiere que el interés por valorar los parques urbanos no solo ha sido constante, sino que ha cobrado renovado interés. Las teorías primarias propuestas en la década de los 1960 siguen vigentes, solo se han presentado refinamientos que permiten solventar el incumplimiento de algunos de los supuestos de los modelos, o que mejoran la validez de estos modelos, y otros que incluyen factores adicionales a los originalmente considerados. En esencia, los esquemas de los modelos permanecen como se concibieron originalmente, no se aprecia un especial dinamismo en su evolución.

El esquema MPH es el más utilizado, sobre todo en países desarrollados. El MPH permite capitalizar las externalidades derivadas de residir dentro del radio de influencia de un parque. En este sentido, el valor de las viviendas parece conservarse en un rango de casi $500 \mathrm{~m}$ en Estados Unidos, mientras que en países con ciudades más densas este rango parece ser mayor, llegando hasta los $650 \mathrm{~m}$. En la literatura se sugiere que la densificación potenciaría el efecto del parque sobre el precio de las viviendas (Dehring y Dunse, 2006). Esta idea parece estar en línea con los porcentajes de incremento del valor de las viviendas, que de 5.6\% que experimentan en Estados Unidos, pasan a valores de $10.53 \%$ y $12.68 \%$ en Asia-Oceanía y Europa, respectivamente. En lo que respecta a Latinoamérica, el número de estudios es aún escaso, lo que impide establecer conclusiones sólidas. En promedio, si una vivienda está localizada dentro de una distancia de $520.22 \mathrm{~m}$ de un parque urbano, su valor de mercado aumenta en 9,68\%. 
En cuanto al MCV, se aprecia que no es utilizado a escala menor, como en parques de barrio o plazas públicas, sino que su adopción se concentra en espacios relativamente grandes donde vale la pena incurrir en un costo para trasladarse y visitarlo. Los estudios de caso en Estados Unidos son prácticamente inexistentes; no obstante, se identificaron algunos en Latinoamérica, sobre a todo a escala periurbana. EI MCV puede ser más pertinente para los casos en los que el traslado dentro de la misma ciudad signifique un gasto importante en relación con el ingreso del visitante, por lo que su uso sería recomendable en este tipo de escenarios.

La VC es el esquema más flexible, ya que permite valorar tanto el uso como el no uso de los parques. Aunque en Estados Unidos no se adopta con mucha frecuencia para dichos propósitos, los resultados encontrados muestran que no es el caso en Europa. De nuevo, este hallazgo puede dar lugar a la idea que en las regiones con ciudades más densas el valor de los parques sería más apreciado y los ejercicios de valoración más frecuentes. Se puede concluir que la VC es más adecuada para adoptarse en valoraciones a escala parque, que es en la que se encontró el mayor número de publicaciones. No obstante la aplicabilidad de la VC en diferentes circunstancias, las publicaciones son reiterativas en afirmar que el instrumento de medición debe ser bien diseñado y que se debe tener especial cuidado durante las entrevistas con los participantes para no obtener respuestas sesgadas, negativas o de protesta (Chen y Hua, 2015; Chen y Qi, 2018; Del Saz-Salazar y Rausell-Köster, 2008).

Este trabajo reveló que la práctica de evaluar económicamente los parques urbanos no solo está vigente, sino que va en franco aumento. En la inmensa mayoría de los estudios de caso presentados fue posible identificar un valor económico positivo. Conocer y ponderar los beneficios económicos de los parques pone de manifiesto su importancia para la sustentabilidad de las ciudades, lo que a la postre hace que se les reconozca como elementos valiosos, incrementando así el número y calidad de estos espacios. El lenguaje del dinero permite también poner en perspectiva la toma de decisiones económica respecto de su mantenimiento y su conservación, además de potenciar su papel como espacio público en la conformación, integración y la cohesión de las sociedades urbanas y de sus imaginarios colectivos.

\section{Referencias}

Abidoye, R. B., y A. P. C. Chan. 2017. "Critical review of hedonic pricing model application in property price appraisal: A case of Nigeria", International Journal of Sustainable Built Environment, 6(1): 250-259. https://doi.org/10.1016/j.ijsbe.2017.02.007

Allen, P. G., T. H. Stevens y T. A. More. 1985. "Measuring the economic value of urban parks: A caution", Leisure Sciences, 7(4): 467-477. https://doi.org/10.1080/01490408509512136

Araque, A., J. Gutiérrez y L. Quenguan. 2017. "El espacio público en la ciudad: Una aproximación desde los precios hedónicos", Sociedad y Economía, 33: 77-98. https://doi.org/10.25100/ sye.v0i33.5625

Arrow, K., R. Solow, P. Portney, E. Leamer, R. Radner y H. Schuman. 1993. Report of the NOAA Panel on Contingent Valuation. researchgate.net. Recuperado de https://www. researchgate.net/profile/Edward Leamer/publication/277297107 Kenneth_Arrow/ links/572a241108ae2efbfdbc1959/Kenneth-Arrow.pdf 
Barnett, C. J. 1985. "An Application of the Hedonic Price Model to the Perth Residential Land Market", Economic Record, March 1985, 61(1): 476-481. https://doi. org/10.1111/i.1475-4932.1985.tb01999.x

Bartczak, A., H. Lindhjem, S. Navrud, M. Zandersen y T. Zylicz. 2008. "Valuing forest recreation on the national level in a transition economy: The case of Poland", Forest Policy and Economics, 10(7-8): 467-472. https://doi.org/10.1016/j.forpol.2008.04.002

Bengochea Morancho, A. 2003. "A hedonic valuation of urban green areas", Landscape and Urban Planning, 66(1): 35-41. https://doi.org/10.1016/S0169-2046(03)00093-8

Biao, Z., X. Gaodi, X. Bin y Z. Canqiang. 2012. "The Effects of Public Green Spaces on Residential Property Value in Beijing", Journal of Resources and Ecology, 3(3): 243-252. https://doi. org/10.5814/i.issn.1674-764x.2012.03.007

Bolitzer, B. y N. R. Netusil. 2000. "The impact of open spaces on property values in Portland, Oregon", Journal of Environmental Management, 59(3): 185-193. https://doi.org/10.1006/ jema.2000.0351

Borja, J. 2011. "Espacio Público y Derecho a La Ciudad. Public space and the right to the city", Viento Sur, 116 (Mayo).

Borzykowski, N., A. Baranzini y D. Maradan. 2018. "Scope Effects in Contingent Valuation: Does the Assumed Statistical Distribution of WTP Matter?", Ecological Economics, 144(February): 319-329. https://doi.org/10.1016/j.ecolecon.2017.09.005

Brander, L. M. y M. J. Koetse. 2011. "The value of urban open space: Meta-analyses of contingent valuation and hedonic pricing results", Journal of Environmental Management, 92(10): 2763-2773. https://doi.org/10.1016/i.jenvman.2011.06.019

Calleja, A., L. Díaz-Balteiro, C. Iglesias-Merchan y M. Soliño. 2017. "Acoustic and economic valuation of soundscape: An application to the 'Retiro' Urban Forest Park", Urban Forestry and Urban Greening, 27(Octuber): 272-278. https://doi.org/10.1016/i.ufug.2017.08.018

Carson, R. T., N. E. Flores, K. M. Martin y J. L. Wright. 1996. "Contingent valuation and revealed preference methodologies: Comparing the estimates for quasi-public goods", Land Economics, 72(1): 80-99. https://doi.org/10.2307/3147159

Carson, R. T., N. E. Flores y N. F. Meade. 2001. "Contingent valuation: Controversies and evidence", Environmental and Resource Economics, 19(2): 173-210. https://doi. org/10.1023/A:1011128332243

Cerda, A., M. Riofrío y F. Vial. (1997). Valoración Contingente para La Creación de la Reserva Shangri-La en la VIII región. Lecturas de Economía, 47: 50-63.

Cesario, F. J. 1976. "Value of Time in Recreation Benefit Studies", Land Economics, 52(1): 32-41. https://doi.org/10.2307/3144984

Chaudhry, P. y V. Tewari. 2006. "A comparison between TCM and CVM in assessing the recreational use value of urban forestry", International Forestry Review, 8(4): 439-448. https://doi.org/10.1505/ifor.8.4.439

Chen, B. y X. Qi. 2018. "Protest response and contingent valuation of an urban forest park in Fuzhou City, China", Urban Forestry and Urban Greening, 29 (January): 68-76. https://doi. org/10.1016/i.ufug.2017.11.005 
Chen, W. Y. y J. Hua. 2015. "Citizens' distrust of government and their protest responses in a contingent valuation study of urban heritage trees in Guangzhou, China", Journal of Environmental Management, 155(15): 40-48. https://doi.org/10.1016/i. jenvman.2015.03.002

Chen, W. Y. y C. Y. Jim. 2008. "Assessment and valuation of the Ecosystem Services provided by urban forests", in Carreiro M.M., YC. Song y J. Wu (eds), Ecology, Planning, and Management of Urban Forests - International Perspectives, 53-83. New York, NY: Springer New York. https://doi.org/10.1007/978-0-387-71425-7 5

Chen, W. Y. y C. Y. Jim. 2010. "Amenities and disamenities: A hedonic analysis of the heterogeneous urban landscape in Shenzhen (China)", Geographical Journal, 176(3): 227-240. https://doi. org/10.1111/i.1475-4959.2010.00358.x

Chen, W. Y. y C. Y. Jim. 2012. "Contingent valuation of ecotourism development in country parks in the urban shadow", International Journal of Sustainable Development and World Ecology, 19(1): 44-53 https://doi.org/10.1080/13504509.2011.588727

Cho, S.-H., C. D. Clark, W. M. Park y S. G. Kim. 2009a. "Spatial and Temporal Variation in the Housing Market Values of Lot Size and Open Space", Land Economics, 85(1): 51-73. https:// doi.org/10.3368/le.85.1.51

Cho, S. H., J. M. Bowker y W. M. Park. 2006. "Measuring the contribution of water and green space amenities to housing values: An application and comparison of spatially weighted hedonic models", Journal of Agricultural and Resource Economics, 31(3): 485-507. Recuperado de https://jareonline.org/articles/measuring-the-contribution-of-water-andgreen-space-amenities-to-housing-values-an-application-and-comparison-of-spatiallyweighted-hedonic-models/

Cho, S. H., S. G. Kim y R. K. Roberts. 2011. "Values of environmental landscape amenities during the 2000-2006 real estate boom and subsequent 2008 recession", Journal of Environmental Planning and Management, 54(1): 71-91. https://doi.org/10.1080/09640568.2010.502760

Cho, S. H., S. G. Kim, R. K. Roberts y S. Jung. 2009b. "Amenity values of spatial configurations of forest landscapes over space and time in the Southern Appalachian Highlands", Ecological Economics, 68(10): 2646-2657. https://doi.org/10.1016/i.ecolecon.2009.04.017

Cho, S. H., T. Kim, R. K. Roberts, C. Hellwinckel, S. G. Kim y B. Wilson. 2014. "Developing an amenity value calculator for urban forest landscapes", Computers, Environment and Urban Systems, 43(January): 34-41. https://doi.org/10.1016/i.compenvurbsys.2013.10.005

Choumert, J. y J. Salanié. 2008. "Provision of urban green spaces: Some insights from economics", Landscape Research, 33(3): 331-345. https://doi.org/10.1080/01426390802045996

Clawson, M. y J. L. Knetsch. 2013. Economics of outdoor recreation (Vol. 3). 328 pp. New York/ London: RFF Press. https://doi.org/10.4324/9781315064215.

Colwell, P. F. y G. Dilmore. 1999. "Who Was First? An Examination of an Early Hedonic Study", Land Economics, 75(4): 620-626. https://doi.org/10.2307/3147070

Conway, D., C. Q. Li, J. Wolch, C. Kahle y M. Jerrett. 2010. "A spatial autocorrelation approach for examining the effects of urban greenspace on residential property values", Journal of Real Estate Finance and Economics, 41(2):150-169. https://doi.org/10.1007/s11146-008-9159-6 
Cook, D., K. Eiríksdóttir, B. Davíđsdóttir y D. M. Kristófersson. 2018. "The contingent valuation study of Heiðmörk, Iceland-Willingness to pay for its preservation". Journal of Environmental Management, 209: 126-138. https://doi.org/10.1016/i.jenvman.2017.12.045

Córdova, A. y J. Martínez-Soto. 2014. "Beneficios de la naturaleza urbana", en Lina Ojeda Revah/ Ileana Espejel (Ed.), Cuando las areas verdes se transforman en paisajes urbanos. La vision de Baja California. (diciembre, pp. 21-50). Tijuana Baja California: El Colegio de la Frontera Norte.

Correll, M. R., J. H. Lillydahl y L. D. Singell. 1978. "The Effects of Greenbelts on Residential Property Values: Some Findings on the Political Economy of Open Space", Land Economics, 54(2): 207-217. https://doi.org/10.2307/3146234

Cristeche, E., y J. A. Penna. 2008. "Métodos de valoración económica de los servicios ambientales", Estudios Socioeconomicos de La Sustentabilidad de Los Sistemas de Producción y Recursos Naturales, (3): 55. https://inta.gob.ar/sites/default/files/script-tmp-metodos doc 03.pdf

Crompton, J. L. 2001. "The Impact of Parks on Property Values: A Review of the Empirical Evidence", Journal of Leisure Research, 33(1): 1-31. https://doi.org/10.1080/00222216.2001.11949928

Crompton, J. L. 2005. "The impact of parks on property values: Empirical evidence from the past two decades in the United States", Managing Leisure, 10(4): 203-218 https://doi. org/10.1080/13606710500348060

Cropper, M. L., L. B. Deck y K. E. McConnell. 1988. "On the Choice of Funtional Form for Hedonic Price Functions", The Review of Economics and Statistics, 70(4): 668. https://doi. org/10.2307/1935831

Darling, A. H. 1973. "Measuring Benefits Generated by Urban Water Parks", Land Economics, 49(1): 22-34. https://doi.org/10.2307/3145326

Davis, R. K. 1963. "Recreation planning as an economic problem". Resource for the Future, 3(2): 239-249. Recuperado de https://core.ac.uk/download/pdf/151602824.pdf

De Frutos Madrazo, P., y S. Esteban Laleona. 2006. El valor de las zonas verdes urbanas. Aplicación del método de los precios hedónicos al mercado inmobiliario de la ciudad de Soria. Soria, España. Recuperado de https://old.aecr.org/web/congresos/2006/ATVI/ATVI-1.pdf

De Frutos Madrazo, P. y Esteban, S. 2009. "Estimación de los beneficios generados por los parques y jardines urbanos a través del método de valoración contingente". Urban Public Economics Review, 10: 13-51.

Dehring, C., y N. Dunse. 2006. "Housing Density and the Effect of Proximity to Public Open Space in Aberdeen, Scotland", Real Estate Economics, 34(4): 553-566. https://doi.org/10.1111/ j.1540-6229.2006.00178.x

Del Saz-Salazar S. y P. Rausell-Köster. 2008. "A Double-Hurdle model of urban green areas valuation: Dealing with zero responses". Landscape and Urban Planning, 84(3-4): 241-251. https://doi.org/10.1016/i.landurbplan.2007.08.008

Del Saz-Salazar, S. y L. García-Menéndez. 2007. "Estimating the non-market benefits of an urban park: Does proximity matter?" Land Use Policy, 24(1): 296-305. https://doi. org/10.1016/i.landusepol.2005.05.011

Dumenu, W. K. 2013. "What are we missing? Economic value of an urban forest in Ghana", Ecosystem Services, 5(September): 137-142.. https://doi.org/10.1016/j.ecoser.2013.07.001 
Engström, G. y A. Gren. 2017. "Capturing the value of green space in urban parks in a sustainable urban planning and design context". Ecology and Society, 22(2). Recuperado de http:// www.jstor.org/stable/26270097

Escobar-Jaramillo, L. A. y L. Ramírez-Zárate. 2009. "Valoración económica de los beneficios sociales del ecoparque urbano Lago de las Garzas en Cali". Ingeniería de Recursos Naturales y Del Ambiente, (8): 93-105., (8): 93-105. Recuperado de http://bibliotecadigital.univalle. edu.co/xmlui/bitstream/handle/10893/2617/paginas 93-105 Revista 8.pdf?sequence=6

Escobar, L. A. y A. Erazo. 2006. "Valoración económica de los servicios ambientales del Bosque de Yotoco: Una estimación comparativa de valoración contingente y coste de viaje", Gestión y Ambiente, 9(1): 25-38.

Ferreira, S. y M. Moro. 2010. "On the use of subjective well-being data for environmental valuation", Environmental and Resource Economics, 46: 249-273. https://doi.org/10.1007/ $\underline{\text { s10640-009-9339-8 }}$

Flores-Xolocotzi, R., M. D. J. González-Guillén y H. M. de los Santos-Posadas. 2010. "Valoración económica del servicio recreativo del parque Hundido de la Ciudad de México", Región y Sociedad, 22(47): 123-144.

Franco, S. F. y J. L. Macdonald. 2016. "Measurement and valuation of urban greenness: Remote sensing and hedonic applications to Lisbon, Portugal", Regional Science and Urban Economics, 72(September): 156-180. https://doi.org/10.1016/j.regsciurbeco.2017.03.002

Friedman, M. y R. D. Friedman. 1962. Capitalism and freedom. Chicago: University of Chicago Press. Gándara, G. 2006. "Valoración económica de los servicios recreativos del Parque Ecológico Chipinque", Working Papers 20064, Escuela de Graduados en Administración Pública y Políticas Públicas, Campus Monterrey. Recuperado de https://ideas.repec.org/p/egb/ wpaper/20064.html

Griliches, Z. 1971. Price indexes and quality change. Harvard University Press. Recuperado de: https://www.hup.harvard.edu/catalog.php?isbn=9780674592582

Gruehn, D. 2008. "Economic Valuation of Ecosystem Services of Urban Open Spaces - Contribution of Urban Green to Life Quality in European Cities". In: Schweppe-Kraft, B. [Ed.]: Ecosystem Services of Natural and Semi-Natural Ecosystems and Ecologically Sound Land Use. BfNSkripten 237: pp. 109-118. Bonn.

Hagerty, J. K., T. H. Stevens y P. G. A. T. More. 1982. "Benefit from urban open space and recreational parks: A case study". Journal of the Northeastern Agriculture Economic Council, XI(1), 1320. https://doi.org/10.1017/S0163548400003125

Halvorsen, R., y H. O. Pollakowski. 1981. "Choice of functional form for hedonic price equations", Journal of Urban Economics, 10(1): 37-49. https://doi.org/10.1016/0094-1190(81)90021-8

Hammer, T. R., R. E. Coughlin y E. T. Horn. 1974. "The Effect of a Large Urban Park on Real Estate Value", Journal of the American Institute of Planners, 40(4): 274-277. https://doi. org/10.1080/01944367408977479

Hanauer, M. M., y J. Reid. 2017. "Valuing urban open space using the travel-cost method and the implications of measurement error", Journal of Environmental Management, 198(2): 50-65. https://doi.org/10.1016/j.jenvman.2017.05.005 
Harnik, P., y B. Welle. 2009. Measuring the Economic Value of a City Park System. The Trust for Public Land. Recuperado de http://cloud.tpl.org/pubs/ccpe-econvalueparks-rpt.pdf

Hatton MacDonald, D., N. D. Crossman, P. Mahmoudi, L. O.Taylor, D. M. Summers y Boxall, P. C. 2010. "The value of public and private green spaces under water restrictions". Landscape and Urban Planning, 95(4): 192-200. https://doi.org/10.1016/j.landurbplan.2010.01.003

Hernández, E. A., M. Jiménez, R. Valdivia y C. Guevara. 2013. Valoración económica del Parque Nacional Xicohténcatl del Estado de Tlaxcala. Puebla Mexico. Recuperado de https:// upaep.mx/micrositios/coloquios/coloquio2013/memorias/Mesa 7 DEySE/Arely Hernandez Sanchez.pdf

Hotelling, H. 1949. "Letter to the national park service", An Economic Study of the Monetary Evaluation of Recreation in the National Parks. US Department of the Interior, National Park Service and Recreational Planning Division, 1949.

Jansson, M. y A. Axel. 2000. "Hedonic Prices for Housing and Adaptation of the RESET Test in non Linear Models. Use of the Box and Cox model to explain housing prices in Catamarca City, Argentina", Revista Semestral Pharos Arte, Ciencia y Cultura, 7(2): 43-59. Recuperado de http://www.redalyc.org/pdf/208/20807205.pdf

Jim, C. Y. y W. Y. Chen. 2006. "Recreation-amenity use and contingent valuation of urban greenspaces in Guangzhou, China". Landscape and Urban Planning, 78(1-2): 81-96. https:// doi.org/10.1016/j.landurbplan.2004.08.008

Jim, C. Y. y W. Y. Chen. 2010. "External effects of neighbourhood parks and landscape elements on high-rise residential value", Land Use Policy, 27(2): 662-670. https://doi.org/10.1016/j. landusepol.2009.08.027

Kitchen, J. W. y W. S. Hendon. 1967. "Land values adjacent to an urban neighborhood park", Land Economics, 43(3): 357-360. https://doi.org/10.2307/3145164

Kong, F. H., H. W.Yin y N. Nakagoshi. 2007. "Using GIS and landscape metrics in the hedonic price modeling of the amenity value of urban green space: A case study in Jinan City, China", Landscape and Urban Planning, 79(3-4): 240-252. https://doi.org/10.1016/j. landurbplan.2006.02.013

Kovacs, K. F. 2012. "Integrating property value and local recreation models to value ecosystem services from regional parks", Landscape and Urban Planning, 108(2-4): 79-90. https://doi. org/10.1016/i.landurbplan.2012.08.002

Lancaster, K. J. 1966. "A New Approach to Consumer Theory", Journal of Political Economy, 74(2): 132. https://doi.org/10.1086/259131

Larqué, B. S., R. Valdivia, F. Islasz y J. L. Romo. 2004. "Valoración económica de los servicios ambientales del bosque del municipio de Ixtapaluca, Estado de México", Revista Internacional de Contaminacion Ambiental, 20(4): 193-202. Recuperado de https://www. redalyc.org/pdf/370/37020406.pdf

Latinopoulos, D., Z. Mallios y P. Latinopoulos. 2016. "Valuing the benefits of an urban park project: A contingent valuation study in Thessaloniki, Greece", Land Use Policy, 55(September): 130141. https://doi.org/10.1016/i.landusepol.2016.03.020

Liebelt, V., S. Bartke y N. Schwarz. 2018. "Hedonic pricing analysis of the influence of urban green spaces onto residential prices: the case of Leipzig, Germany". European Planning Studies, 26: 133-157. https://doi.org/10.1080/09654313.2017.1376314 
Liu, S., y D. Hite. 2013. "Measuring the Effect of Green Space on Property Value: An Application of the Hedonic Spatial Quantile Regression", In Southern Agricultural Economic Association Annual Meeting. 2013 Annual Meeting. February 2-5, 2013, Orlando, Florida.

Lo, A. Y., y C. Y. Jim. 2010. "Willingness of residents to pay and motives for conservation of urban green spaces in the compact city of Hong Kong", Urban Forestry and Urban Greening, 9(2): 113-120. https://doi.org/10.1016/j.ufug.2010.01.001

Lockwood, M., y K. Tracy. 1995. "Nonmarket Economic Valuation of an Urban Recreation Park", Journal of Leisure Research. 27(2):155-167. https://doi.org/http://dx.doi. org/10.1108/17506200710779521

López-Mosquera, N., T. García y R. Barrena. 2014. "An extension of the Theory of Planned Behavior to predict willingness to pay for the conservation of an urban park". Journal of Environmental Management, 135(15): 91-99. https://doi.org/10.1016/j.jenvman.2014.01.019

López-Mosquera, N., y M. Sánchez. 2011. "The influence of personal values in the economic-use valuation of peri-urban green spaces: An application of the means-end chain theory", Tourism Management, 32(4): 875-889. https://doi.org/10.1016/i.tourman.2010.08.003

López-Mosquera, N., y M. Sánchez. 2012. "Theory of Planned Behavior and the Value-Belief-Norm Theory explaining willingness to pay for a suburban park", Journal of Environmental Management, 113(30) 251-262. https://doi.org/10.1016/i.jenvman.2012.08.029

Luttik, J. 2000. "The value of trees, water and open space as reflected by house prices in the Netherlands", Landscape and Urban Planning, 48(3-4): 161-167. https://doi.org/10.1016/ S0169-2046(00)00039-6

Lutzenhiser, M., y N. R. Netusil. 2001. "The effect of open spaces on a home's sale price", Contemporary Economic Policy, 19(3): 291-298. https://doi.org/10.1093/cep/19.3.291

Majumdar, S., J. Deng, Y. Zhang y C. Pierskalla. 2011. "Using contingent valuation to estimate the willingness of tourists to pay for urban forests: A study in Savannah, Georgia", Urban Forestry and Urban Greening, 10(4): 275-280. https://doi.org/10.1016/i.ufug.2011.07.006 Martínez-Cruz, A. L. 2005. "El valor consuntivo del Desierto de los Leones". Gaceta Ecológica, 75: 51-64.

Martínez-Cruz, A., y J. Sainz. 2017. "El valor de dos espacios recreativos periurbanos en la Ciudad de México. The value of two recreative periurban spaces in Mexico City". El Trimestre Económico, 84(336): 805-816. https://doi.org/10.20430/ete.v84i336.607

Mayor, K., S. Lyons, D. Duffy y R. S. J. Tol. 2009. "A hedonic analysis of the value of parks and green spaces in the Dublin area". Papers WP331, Economic and Social Research Institute.

McLeod, P. B. 1984. "The demand for local amenity: an hedonic price analysis", Environment and Planning A: Economy and Space, 16(3): 389-400. https://doi.org/10.1068/a160389

Morales, D. J. 1980. "The contribution of trees to residential property value. Journal of Arboriculture, 6(11): 305-308.

More, T. A., T. Stevens y P. G. Allen. 1988. "Valuation of urban parks". Landscape and Urban Planning, 15(1-2): 139-152. https://doi.org/10.1016/0169-2046(88)90022-9

Moreno Murrieta, Ramsés y E. Alvarado Lagunas. 2011. "El entorno social y su impacto en el precio de la vivienda: Un análisis de precios hedónicos en el Area Metropolitana de Monterrey". Trayectorias, 14(33-34): 131-147. Recuperado de http://www.redalyc.org/ pdf/607/60724509007.pdf 
Muñoz, D., Villeda, M., Rivera, P., Reyna, M., Osornio, Á., y Martínez-Cruz, A. L. 2020. "Stated benefits form air quality improvememnt thtoug urban afforestation in an arid city - A contingent valuation in Mexicali, Baja California, México". Urban Forestry \& Urban Greening, In-press., 55(November). https://doi.org/https://doi.org/10.1016/i.ufug.2020.126854

Neckel, A., Lima, J., Pol, P., Kujawa, H., Araldi, J., y Pacheco, E. 2020. "Estimation of the economic value of urban parks in Brazil, te case of the City of Passo Fundo". Journal of Cleaner Proeduction, 264(10 agosto 2020). https://doi.org/https://doi.org/10.1016/j. jclepro.2020.121369

Nielsen, A. B., S. B. Olsen y T. Lundhede. 2007. "An economic valuation of the recreational benefits associated with nature-based forest management practices", Landscape and Urban Planning, 80(1-2): 63-71. https://doi.org/10.1016/i.landurbplan.2006.06.003

Noor, N. M., M. Z. Asmawi y A. Abdullah. 2015. "Sustainable Urban Regeneration: GIS and Hedonic Pricing Method in Determining the Value of Green Space in Housing Area", Procedia - Social and Behavioral Sciences, 170(27): 669-679. https://doi.org/10.1016/j.sbspro.2015.01.069

Osorio, J. D., y F. Correa. 2004. "Valoracion Economica de Costos Ambientales: marco conceptual y metodos de estimacion", Semestre Economico, 7(13): 159-193. Recuperado de https:// revistas.udem.edu.co/index.php/economico/article/view/1141

Panduro, T. E., y K. L. Veie. 2013. "Classification and valuation of urban green spaces-A hedonic house price valuation", Landscape and Urban Planning, 120(December): 119-128. https:// doi.org/10.1016/j.landurbplan.2013.08.009

Penagos-Concha, A. M. 2005. "¿Estan los bogotanos interesados en vivir cerca de un parque urbano?" Sociedad y Economía, 9: 81-104. Recuperado de http://www.redalyc.org/articulo. oa?id=99620854005

Perino, G., B. Andrews, A. Kontoleon y I. Bateman. 2014. "The Value of Urban Green Space in Britain: A Methodological Framework for Spatially Referenced Benefit Transfer", Environmental and Resource Economics, 57(2): 251-272. https://doi.org/10.1007/s10640$\underline{013-9665-8}$

Platania, M. y M. Rizzo. 2018. "Willingness to pay for protected areas: A case of Etna Park", Ecological Indicators, 93(Octuber): 201-206. https://doi.org/10.1016/i.ecolind.2018.04.079

Poudyal, N. C., D. G. Hodges y C. D. Merrett. 2009. "A hedonic analysis of the demand for and benefits of urban recreation parks", Land Use Policy, 26(4): 975-983. https://doi. org/10.1016/i.landusepol.2008.11.008

Rivas, A. y J. Ramoni. 2002. "Valoración contingente aplicada al Parque Metropolitano Albarregas (Mérida, Venezuela)", Economía, 18(17-18): 119-133. Recuperado de https:// econpapers.repec.org/article/ulaeconom/v_3a26-27_3ay_3a2001-2002_3ai_3a1718 3ap 3a119-133.htm

Romero, J. P. y J. J. Vargas. 2016. "Valoración ambiental de las zonas verdes de una urbanización en Bogotá, Colombia, con el método de precios hedónicos". Semestre Económico 19(39):1330. https://doi.org/10.22395/seec.v19n39a1

Rosen, S. 1974. "Hedonic Prices and Implicit Markets: Product Differentiation in Pure Competition", Journal of Political Economy, 82(1): 34-55. https://doi.org/10.1086/260169 
Sánchez, J. M. 2008. "Valoración contingente y costo de viaje aplicados al área recreativa laguna de Mucubají", Economía, 23(6): 119-150. Recuperado de http://revencyt.ula.ve/storage/ repo/ArchivoDocumento/econo/n26/articulo5.pdf

Sander, H. A. y R. G. Haight. 2012. "Estimating the economic value of cultural ecosystem services in an urbanizing area using hedonic pricing", Journal of Environmental Management, 113(December): 194-205. https://doi.org/10.1016/j.jenvman.2012.08.031

Sander, H. A. y S. Polasky. 2009. "The value of views and open space: Estimates from a hedonic pricing model for Ramsey County, Minnesota, USA", Land Use Policy, 26(3): 837-845. https:// doi.org/10.1016/i.landusepol.2008.10.009

Sander, H., S. Polasky y R. G. Haight. 2010. "The value of urban tree cover: A hedonic property price model in Ramsey and Dakota Counties, Minnesota, USA", Ecological Economics 69(8): 1646-1656. https://doi.org/10.1016/i.ecolecon.2010.03.011

Saphores, J.-D. y W. Li. 2012. Landscape and Urban Planning Estimating the value of urban green areas: A hedonic pricing analysis of the single family housing market in Los Angeles , CA. Landscape and Urban Planning, 104(3-4): 373-387. https://doi.org/10.1016/i. landurbplan.2011.11.012

Schläpfer, F., F. Waltert, L. Segura y F. Kienast. 2015. "Valuation of landscape amenities: A hedonic pricing analysis of housing rents in urban, suburban and periurban Switzerland". Landscape and Urban Planning, 141(September): 24-40. https://doi.org/10.1016/i. landurbplan.2015.04.007

Sepulveda, R. D. 2008. "Economic valuation of the recreational use of "Ronda del Sinú" park in Monteria, Colombia", Semestre Economico, 11(22): 67-90. Recuperado de https://core. ac.uk/download/pdf/6302474.pdf

Silva, A. 2006. Imaginarios Urbanos. (A. Editores, Ed.) (5a.). Bogota. Colombia: Editorial Nomos.

Sirina, N., A. Hua y J. Gobert. 2017. "What factors influence the value of an urban park within a medium-sized French conurbation?", Urban Forestry and Urban Greening, 24(May): 4554. https://doi.org/10.1016/j.ufug.2017.03.021

Tajima, K. 2003. "New estimates of the demand for urban green space: Implications for valuing the environmental benefits of Boston's big dig project. Journal of Urban Affairs, 25(5): 641655. https://doi.org/10.1111/i.1467-9906.2003.00006.x

Tameko, A. M., H. P. Donfouet y F. Sikod. 2011. "The Economic Valuation of Improved Urban Parks: A Case Study of Warda Park", Journal of Sustainable Development, 4(1): 271-281. https:// doi.org/10.5539/isd.v4n1p271

Thayer, M. A. 1981. "Contingent valuation techniques for assessing environmental impacts:

Further evidence". Journal of Environmental Economics and Management, 8(1): 27-44 https://doi.org/10.1016/0095-0696(81)90055-3

Tibesigwa, B., Herbert, N., y Lokina, R. 2020. "Valuing recreational ecosystem services in developing cities: The case of urban parks in Dar es Salaam, Tanzania". Cities, 106 (November). https:// doi.org/https://doi.org/10.1016/i.cities.2020.102853

Tietenberg, T., y L. Lewis. 2012. Environmental and Natural Resource Economics 9th Edition (9th ed.). Pearson Education, Inc. Recuperado de https://www.pearson.com/uk/educators/ higher-education-educators/program/Tietenberg-Environmental-Natural-ResourcesEconomics-Pearson-New-International-Edition-9th-Edition/PGM1052077.html 
Troy, A., y J. M. Grove. 2008. "Property values, parks, and crime: A hedonic analysis in Baltimore, MD". Landscape and Urban Planning, 87(3): 237-245. https://doi.org/10.1016/i. landurbplan.2008.06.005

Tyrväinen, L. 1997. "The amenity value of the urban forest: an application of the hedonic pricing method", Landscape and Urban Planning, 37(3-4): 211-222. https://doi.org/10.1016/ S0169-2046(97)80005-9

Tyrväinen, L. y A. Miettinen. 2000. "Property prices and urban forest amenities", Journal of Environmental Economics and Management, 39(2): 205-223. https://doi.org/10.1006/ jeem.1999.1097

Tyrväinen, L. y H. Väänänen. 1998. "The economic value of urban forest amenities: An application of the contingent valuation method", Landscape and Urban Planning, 43(1-3): 105-118. https://doi.org/10.1016/S0169-2046(98)00103-0

Urteaga, E. 2013. "La teorìa del capital social de Robert Putnam: Originalidad y carencias", Reflexión Política, 15(29): 44-60. Recuperado de http://www.redalyc.org/articulo. oa?id=11028415005

Varela, J. M. L., M. Börjesson y A. Daly. 2018. "Quantifying errors in travel time and cost byç latent variables", Transportation Research Part B: Methodological, 117: 520-541. https:// doi.org/10.1016/J.TRB.2018.09.010

Vargas, A., y P. Roldán. 2017. "Ni muy cerca ni muy lejos: parques urbanos y bienestar subjetivo en la ciudad de Barranquilla, Colombia". Lecturas de Economía, 88(Enero-Junio): 183-205. https://doi.org/10.17533/udea.le.n88a06

Weigher, J. C., y R. H. Zerbst. 1973. "The Externalities of Neighborhood Parks : An Empirical Investigation", Land Economics, 49(1): 99-105. https://doi.org/10.2307/3145337

Willis, K. G., y J. F. Benson. 1989. "Recreational values of forests", Forestry, 62(2): 93-110. https:// doi.org/10.1093/forestry/62.2.93

Willis, K. G., y G. D. Garrod. 1991. "An individual travel-cost methid of evaluating forest recreation". Journal of Agricultural Economics, 42(1): 33-42. https://doi.org/10.1111/j.1477-9552.1991. tb00330.x

Wilson, E. O. 1984. Biophilia. Harvard. Press, Cambridge (Mass.).

Xu, F., Wang, Y., Xiang, N., Tian, J., y Chen, L. 2020. "Uncovering the willingness-to-pay for urban green space conservation: A survey of the capital area in China". Resources, Conservation and Recycling, 162, 105053. https://doi.org/10.1016/i.resconrec.2020.105053 